\title{
Integration and bioactivity of hydroxyapatite grown on carbon
}

\section{nanotubes and graphene oxide}

\author{
J. David Núñez ${ }^{\mathrm{a}, \mathrm{b}}$, Ana M. Benito ${ }^{\mathrm{a}}$, Ramón González ${ }^{\mathrm{c}}$ Javier Aragón $^{\mathrm{d}}$, Raul Arenal ${ }^{\mathrm{e}, \mathrm{f}}$, \\ and Wolfgang K. Maser ${ }^{\mathrm{a} *}$ \\ ${ }^{a}$ Instituto de Carboquímica ICB-CSIC, E-50018 Zaragoza, Spain. \\ ${ }^{\mathrm{b}}$ Centro de Estudios Avanzados de Cuba, La Habana, Cuba. \\ ${ }^{\mathrm{c}}$ Centro de Neurociencias de Cuba, La Habana, 10600, Cuba. \\ ${ }^{d}$ Centro Nacional de Investigaciones Científicas, La Habana, 10600, Cuba. \\ ${ }^{\mathrm{e}}$ Laboratorio de Microscopias Avanzadas (LMA)-Instituto de Nanociencia de Aragon \\ (INA), Univ. Zaragoza, E-50018 Zaragoza, Spain \\ ${ }^{\mathrm{f}}$ Fundación ARAID, E-50018 Zaragoza, Spain
}

\begin{abstract}
We report on the growth of non-stoichiometric nanocrystalline hydroxyapatite (nHAp) with a composition similar to natural bone by a wet-chemical in-situ precipitation route on carbon nanotubes (CNTs) with different degrees of oxygen functionalities and on graphene oxide (GO). Both, functionalization degree and morphology of CNTs and GO appear as critical parameters controlling the shape and crystallinity of the selfassembled nHAp nanoparticles in the corresponding composite materials. Crystalline nHAp nanoparticles with rod-like morphology were achieved for moderately oxidized
\end{abstract}

*Corresponding author. Tel: +34-976-733977. E-mail: wmaser@icb.csic.es (Wolfgang K. Maser) 
CNTs and for GO. On CNTs these grow along the CNT's axis while on GO they form a compact layer. In both cases enhanced nHAp integration onto the respective carbon support is obtained. In vitro bioactivity of the prepared composites exhibits a fast apatite biomineralization process, induced by the presence of nHAp. Depending on its crystalline size and dispersion degree remineralization of the apatite nanoparticles takes place through the inclusion of $\mathrm{PO}_{4}{ }^{3-}$ and $\mathrm{CO}_{3}{ }^{2-}$ by ion exchange. Importantly, during the stage of bioactivity the integration degree of nHAp nanoparticles on the carbon nanostructures alters with time, evidencing the potential of GO as valuable bioceramic support material.

\section{Introduction}

Hydroxyapatite (HAp) is a bioceramic material that exhibits an excellent biocompatibility and osteogenic potential to promote tissue adhesion and bone growth. Currently, it is the material of choice in dental and orthopedic surgery to fill bone defects and to coat metallic implant surfaces to improve implant integration with host bone $[1,2]$. The composition of $\mathrm{HAp}, \mathrm{Ca}_{10}\left(\mathrm{PO}_{4}\right)_{6}(\mathrm{OH})_{2}$, closely resembles the one of the mineral component of native bone, i.e. biological apatite, a calcium deficient, carbonate-substituted non-stoichiometric hydroxyapatite [3]. A nanocrystalline structure and a rod-like morphology are two further distinct features that contribute to its unique biological functionality under physiological conditions [3]. Thus current research efforts focus on the synthesis of nanosized HAp paralleling natural bone minerals as best choice for bone replacement and regeneration [1]. However, intrinsic brittleness, low wear resistance, and low fracture toughness still limit the use of HAp in load-bearing and long-term implant applications $[2,4,5]$. Mechanical performance of HAp can be 
improved by incorporating second phase reinforcements such as ceramics [6-8] and polymers [9-11].

Recently, carbon nanotubes (CNTs) have attracted great attention as reinforcing phase as documented in reviews by A.A. White [4] and more recently by D. Lahiri [12]. Their unique nanoscale dimensions, high surface area, low density and exceptionally high strength and stiffness $[13,14]$ offer effective load transfer possibilities to the HAp matrix. Various reports on CNT-HAp composite coatings show enhanced strength and fracture toughness, as well as reduced wear resistance [15-18]. Accompanied by favorable osteoblast cell proliferation properties $[19,20]$. These composites are of promise as coating for load-bearing orthopaedic implants. Concerns about adverse cytotoxic effects of CNTs itself may be mitigated by their chemical functionalization [21]. Indeed, it has been shown that oxidized CNTs are not cytotoxic, but accelerate osteoblast proliferation and bone formation, and thus can be explored as suitable scaffold material for biomedical applications [22, 23]. Graphene, as the parent twodimensional structure of CNTs exhibits a similar set of unique properties [24] and is currently explored as alternative reinforcement phase $[25,26]$. As for the case of carbon nanotubes the oxidized form of graphene, i.e. graphene oxide $(\mathrm{GO})$ is biocompatible [27] and biostable [28] and of interest as platform for biomedical applications [29].

The oxidized forms of CNTs and GO are of special interest when wet-chemical synthesis approaches are employed to achieve nanosized hydroxyapatite (nHAp) [1, 3] and respective reinforced HAp composite materials [30-40]. The functional oxygen groups on their surfaces contribute to address the key issues of homogeneous dispersion and interfacial bonding $[4,5]$ and also favour the growth of nanosized hydroxyapatite (nHAp), which in turn positively affects biocompatibility and bioactivity of the corresponding nanocomposites [3]. While biomimetic and hydrothermal approaches can 
be quite time-consuming and complex only few articles report on the more time- and cost-effective and up-scalable in-situ precipitation methods for the preparation of HApCNT/GO composites. By simply using oxidized CNTs [30, 31] or graphene oxide [36] nanocrystalline HAp platelets or spindles with improved interfacial bonding were obtained resulting in composites exhibiting enhanced mechanical properties and improved biocompatibility. First reports on CNTs [30, 31, 34, 35, 41] and graphene oxide $[36,40]$ show that oxygen functionalities can act as effective nucleation sites for precipitating $\mathrm{Ca}^{2+}$ ions and thus influence on the subsequent crystallization and growth process of hydroxyapatite, as well as the interfacial bonding to the support [5]. However, it also was shown that HAp nanorods could grow on reduced graphene oxide, which should lack oxygen functionality, simply through tuning the precipitation condition itself [37]. This is an intriguing situation, and accompanied by the lack of information on the bioactivity behaviour of the developed composites, reveals the need for systematically elucidating the influence of type and density of oxygen functionalities of a given type of carbon nanomaterial on the self-assembled growth of HAp, its integration onto the support, as well as the effect on the bioactivity of the respective composites.

The present work aims to shine more light on these important issues. For this purpose we employed CNTs exhibiting different oxidation degrees as well as GO in a timeefficient wet-chemical in-situ precipitation method directed towards the growth of nonstoichiometric nanocrystalline hydroxyapatite-carbon nanotube/graphene oxide (nHApCNT/GO) nanocomposites. Morphology, crystalline size, composition and interfacial bonding of nHAp to the respective nanocarbon support surface were assessed by transmission electron microscopy (TEM), powder X-ray diffraction (XRD), and X-ray photoelectron spectroscopy (XPS). Furthermore, the in-vitro bioactivity of the 
nanocomposites was studied. Its effect on composition, structure and integration at different stages of the biomineralization process was analyzed.

\section{Materials and methods}

\subsection{Materials}

Pristine carbon nanotubes (Nanocyl ${ }^{\mathrm{TM}}$ NC7000 multi-walled carbon nanotubes, $90 \%$ carbon purity) were purchased from Nanocyl Co., Belgium. All other chemical reagents used in this study were acquired from Aldrich (Sigma-Aldrich Co. St. Louis, MO): graphite powder (ref. number 332491), $\mathrm{HNO}_{3}, \mathrm{NaNO}_{3}, \mathrm{H}_{2} \mathrm{SO}_{4}, \mathrm{KMnO}_{4}, \mathrm{NH}_{4} \mathrm{OH}, \mathrm{CaCO}_{3}$, $\mathrm{H}_{3} \mathrm{PO}_{4}$. Simulated Body Fluid (shortly SBF): $\mathrm{NaCl}, \mathrm{NaHCO}_{3}, \mathrm{KCl}, \mathrm{K}_{2} \mathrm{HPO}_{4} \cdot 3 \mathrm{H}_{2} \mathrm{O}$, $\mathrm{MgCl}_{2} \cdot 6 \mathrm{H}_{2} \mathrm{O}, \mathrm{CaCl}_{2}, \mathrm{Na}_{2} \mathrm{SO}_{4}, \mathrm{HCl}$ and tris(hydroxymethyl)aminomethane (shortly TRIS): $\left(\mathrm{HOCH}_{2}\right)_{3} \mathrm{CNH}_{2}$.

\subsection{Preparation of oxidized carbon nanotubes}

Oxidized carbon nanotubes (oCNTs) with a higher and lower oxidation degree were prepared by refluxing pristine carbon nanotubes (pCNTs) in $9.5 \mathrm{M} \mathrm{HNO}_{3}$ for times of 24 $\mathrm{h}$ and $18 \mathrm{~h}$, respectively. The resulting oxidized carbon nanotubes (oCNTs) were filtered, repeatedly washed with distilled water until neutral $\mathrm{pH}$ was achieved, and dried at $80{ }^{\circ} \mathrm{C}$ under vacuum for $12 \mathrm{~h}$. The samples obtained at reflux times of $24 \mathrm{~h}$ and $18 \mathrm{~h}$ are labelled oCNT1 and oCNT2, respectively.

\subsection{Preparation of graphene oxide}

Graphene oxide (GO) was synthesized in a two-step approach: First, graphite oxide was prepared using a modified Hummers' method from graphite powder by oxidation with $\mathrm{NaNO}_{3}, \mathrm{H}_{2} \mathrm{SO}_{4}$ and $\mathrm{KMnO}_{4}$ in an ice bath as reported elsewhere [42-44] In brief, 170 mL of concentrated $\mathrm{H}_{2} \mathrm{SO}_{4}$ was added to a mixture of graphite flakes $(5.0 \mathrm{~g})$ and $\mathrm{NaNO}_{3}$ 
$(3.75 \mathrm{~g})$. The mixture was cooled in an ice bath, and stirred for thirty minutes. $\mathrm{KMnO}_{4}$

(25 g) was slowly added and stirred for another thirty minutes. The reaction was then warmed to $35{ }^{\circ} \mathrm{C}$, and stirred for two more hours. Water $(250 \mathrm{~mL})$ was slowly added and then $30 \% \mathrm{H}_{2} \mathrm{O}_{2}(20 \mathrm{~mL})$. The mixture was stirred for an hour, filtered, washed with $400 \mathrm{~mL}$ of $\mathrm{HCl}: \mathrm{H}_{2} \mathrm{O}$ (1:10), and dried. Second, $15 \mathrm{mg}$ of the obtained graphite oxide powder was dispersed in $100 \mathrm{~mL}$ of concentrated ammonia. Ultrasonication in a bath sonicator for $1 \mathrm{~h}$ results in a homogeneous and stable brown-colored dispersion indicating the exfoliation of graphite oxide powder into flakes of graphene oxide in aqueous solvents $[44,45]$. In order to achieve compatibility with the synthesis process of hydroxyapatite, in this work ammonia was used instead of water.

\subsection{Synthesis of non-stoichiometric nanocrystalline hydroxyapatite ( $n H A p)$}

Non-stoichiometric nanocrystalline hydroxyapatite (nHAp) was prepared by a rapid wet-chemical precipitation route. Briefly, $2 \mathrm{~g}$ of $\mathrm{CaCO}_{3}$ was dissolved under magnetic stirring in $100 \mathrm{ml}$ of $\mathrm{H}_{3} \mathrm{PO}_{4}(0.3 \mathrm{M}$ in distilled water $)$. The resulting saturated dispersion was filtered and a colorless calcium dihydrogen phosphate precursor solution was obtained. This solution was added drop-wise to $100 \mathrm{ml}$ of concentrated $\mathrm{NH}_{4} \mathrm{OH}$. Applying a continuous vigorous stirring at room temperature and keeping the reaction $\mathrm{pH}$ above 10 to avoid hydroxyapatite dissolution a precipitate was slowly formed on a time-scale of about 30 minutes. The precipitate was filtered and washed with distilled water repeatedly until neutral $\mathrm{pH}$. Subsequently, the remaining white solid was dried at $110{ }^{\circ} \mathrm{C}$ under vacuum overnight.

2.5. Synthesis of composites based on nHAp and different types of carbon nanomaterials 
Similarly to the procedure described in section 2.4, nHAp composites were prepared by previously dispersing the carbon nanomaterial in the ammonia solution before the addition of the precursor solution. In a typical experiment, $15 \mathrm{mg}$ of pCNT, oCNT1, and oCNT2, respectively, was bath-sonicated in $100 \mathrm{ml}$ of concentrated ammonia solution during 30 min achieving homogeneous dispersions. In the case of GO, the as produced stable GO-ammonia dispersion (15 mg/100mL) was used (see Section 2.3). This resulted in four types of composites, namely nHAp-pCNT (nHAp supported on pCNT), nHAp-oCNT1 (nHAp supported on oCNT1), nHAp-oCNT2 (nHAp supported on oCNT2), and nHAp-GO (nHAp supported on GO).

\subsection{In vitro bioactivity assays}

In vitro bioactivity assays were performed by exposing the prepared composite materials to simulated body fluid $(\mathrm{SBF})$, an inorganic physiologic solution having a similar composition of human plasma without organic components [46]. The SBF composition was prepared according to ISO norm 23317 [47] ensuring that bioactivity tests are carried out under standardized condition mimicking the mineralization processes of bone. Also the further experimental protocol followed ISO norm 23317. In detail, the respective samples, i.e. $10 \mathrm{mg}$ of pCNT (control test 1), oCNT2 (control test 2), nHAp-oCNT1, nHAp-oCNT2 and nHAp-GO were dipped into a vial containing 10 $\mathrm{ml}$ of SBF. Samples remained immersed for 1 to 4 weeks in SBF solution at $37{ }^{\circ} \mathrm{C}$. Afterwards, the samples were filtered, gently rinsed with distilled water, and dried at $110^{\circ} \mathrm{C}$ under vacuum overnight. In addition, GO-paper (control test 3), RGO-paper (control test 4) were also tested under the same condition to evaluate the bioactivity of GO with respect to nHAp-GO (see Suppl. Data).

\subsection{Characterization}


Morphology and particle size of the nHAp composites were studied by transmission electron microscopy (TEM) using a JEOL JEM-2000FX II microscope operated at $200 \mathrm{kV}$ and a high resolution Cs-corrected FEI-Titan-Cube microscope working at 80 $\mathrm{kV}$. Samples were dispersed in ethanol in ultrasound bath for a few minutes and a drop of the suspension was placed onto a copper grid coated with carbon film. Structural analyses were performed by X-ray powder diffraction using a Bruker D8 Advance equipped with a Cu-tube $\left(\lambda_{\mathrm{CuK \alpha}}=0.154059 \mathrm{~nm}\right)$ operating at a voltage of $40 \mathrm{kV}$ and a current of $40 \mathrm{~mA}$. Measurements were carried out from $5^{\circ}$ to $80^{\circ}(2 \theta)$ with a step angle of $0.05^{\circ}$. Peak position and indexing was performed using Bruker TOPAS and EVA software packets applying ICDD file card 24-0033 for hydroxyapatite. Mean crystallite size $X_{\mathrm{s}}$ was calculated using Scherrer's equation: $X_{\mathrm{s}}=0.9 \cdot \lambda_{\mathrm{CuK \alpha}} / \beta \cdot \cos (\theta)$, where $\beta$ is the full width of the diffraction peak at half of its maximum intensity (FWHM) in [rad]. Crystal structure modelling was carried out using VESTA programme v. 2.1.6 (2011) applying database code AMSCD 0001257 for Ca5P3O13H, Hexagonal 176, P63/m, a = $\mathrm{b}=0.94166 \mathrm{~nm}, \mathrm{c}=0.6845 \mathrm{~nm}, \alpha=\beta, \gamma=120^{\circ}$. Chemical composition was analysed by X-ray photoelectron spectroscopy (XPS) carried out on an ESCAPlus Omicron spectrometer using a monochromatized Mg X-ray source (1253.6 eV). Data were analyzed using CasaXPS software packet.

\section{Results and discussion}

The growth of hydroxyapatite on the various carbon nanomaterials used as support for forming corresponding composites was carried out following an in-situ wet-chemical synthesis strategy (Figure 1). Its base is the chemical precipitation of calcium and phosphate ions from a saturated precursor dissolution induced by a rapid change in $\mathrm{pH}$ upon dropwise addition to a highly concentrated ammonia solution. Nucleation and growth leads to the self-assembly of non-stoichiometric hydroxyapatite. When carbon 
nanomaterials are dispersed in the ammonia solution the formation of hydroxyapatite takes place on the support and corresponding composites are obtained.

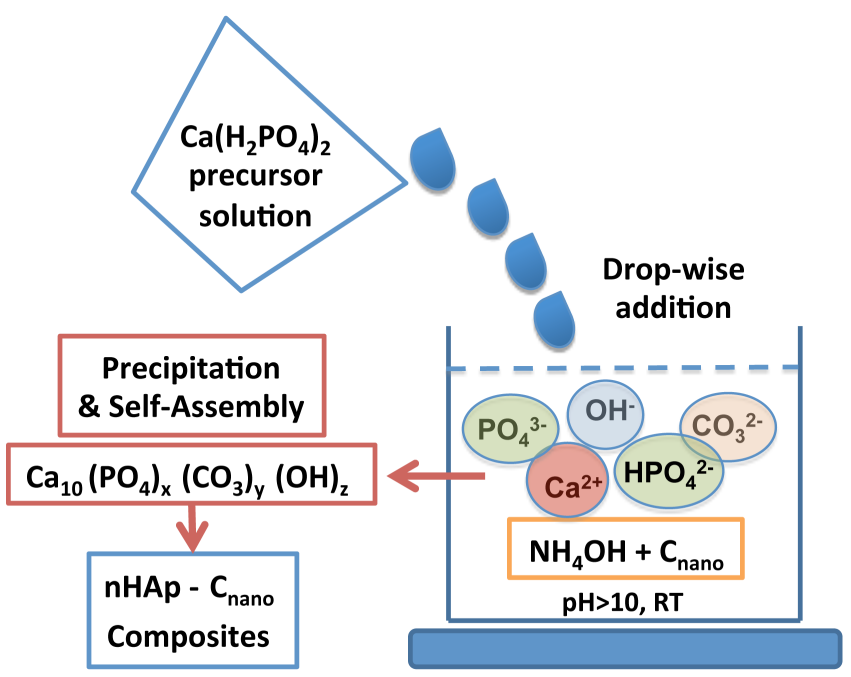

Fig. 1 - Scheme of the wet-chemical synthesis process for the preparation of nHAp$\mathrm{C}_{\text {nano }}$ composites. $\mathrm{C}_{\text {nano }}$ denotes the different forms of carbon nanomaterials used as support, i.e. pristine carbon nanotubes, oxidized carbon nanotubes and graphene oxide.

Oxygen functionalities are thought to act as effective nucleation sites for precipitating $\mathrm{Ca}^{2+}$ ions affecting the subsequent phosphate precipitation and the resultant hydroxyapatite cyrstallization and growth process [30, 31, 34, 36, 40, 41]. However, systematic studies on the influence of oxidation degree and the type and morphology of the carbon nanomaterial on the growth of hydroxyapatite, its crystalline structure, and its support integration are largely missing. Therefore, various types of carbon nanomaterials, i.e. carbon nanotubes with different oxidation degrees as well as graphene oxide were prepared and used as support in the synthesis process. Structure, morphology and chemical characteristics of the starting carbon nanomaterials are presented in Section 3.1. The corresponding hydroxyapatite composite materials are described in Section 3.2. Here emphasis lies on the influence of the carbon material and 
its oxygen functionalities on the growth of hydroxyapatite and its integration on the support. The bioactivity behaviour of the various hydroxyapatite composites is featured in Section 3.3.

\subsection{Carbon nanomaterials employed: Structure, morphology and oxygen functionalities}

Transmission electron microscopy (TEM) images (Figure 2) provide information on the essential structural and morphological features of the carbon nanomaterials employed as support for the growth of hydroxyapatite. Pristine carbon nanotubes (pCNTs) show the typical characteristics of CCVD grown thin multi-wall carbon nanotubes (Figure 2a,b). These exhibit an average external diameter of about $10 \mathrm{~nm}, 8$ graphitic layers around an internal inner core of about $5 \mathrm{~nm}$ in diameter, and lengths in the order of $1 \mu \mathrm{m}$. The CNTs are curved and highly entangled. The outer walls reveal regions with defects and/or depositions of amorphous carbon. This is in agreement with results from Raman spectroscopy and thermogravimetric analysis (TGA) (see Supporting Data). The oxidation treatment affects the structural characteristics of the sidewalls of CNTs. However, structural integrity is maintained even in case of the heavily functionalized oCNT1 sample (Figure 2c). Graphene oxide (GO) flakes produced by the exfoliation of graphite oxide typically have lateral dimensions in the order of $1 \mu \mathrm{m}$ and reveal an overall planar morphology as shown by TEM images (Figures $2 \mathrm{~d}$ ). The observed wrinkles and folds most likely are related to the TEM preparation/deposition process and do not necessarily reflect the intrinsic nature of GO. Please note that the exfoliated GO sheets are not single-layered, but comprise a small number of layers, as explained in section 3.2 . 

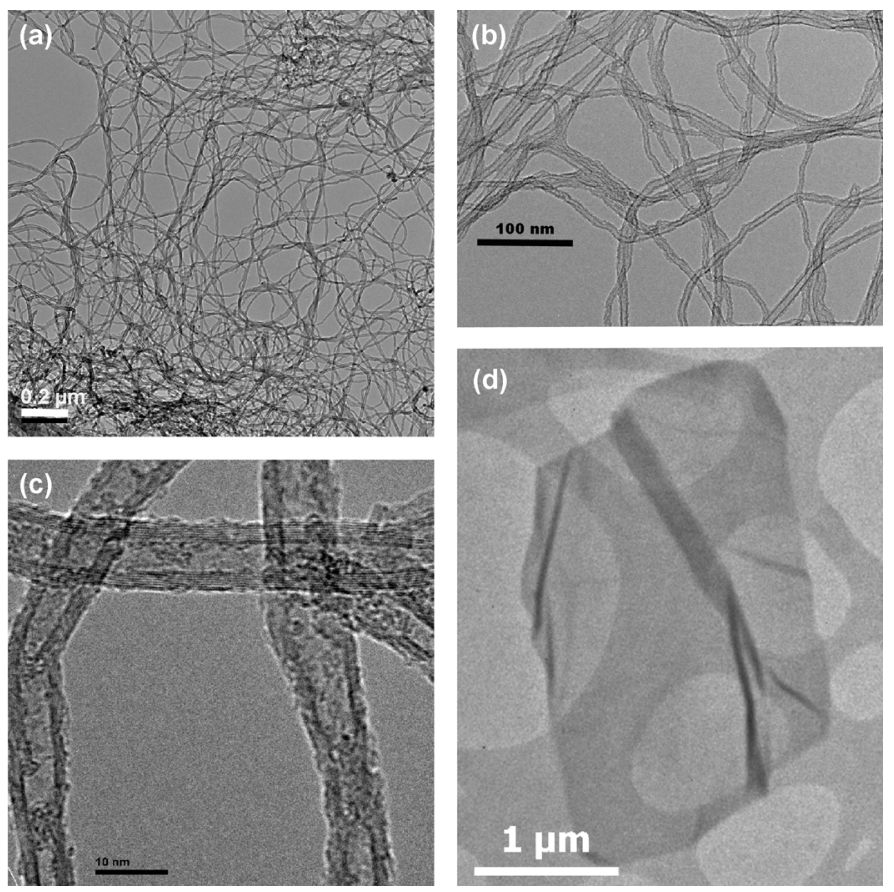

Fig. 2 - TEM images of carbon nanomaterials used as support for nHAps. (a,b) pCNTs, (c) heavily oxidized oCNT1 at high magnification, (d) graphene oxide flakes obtained by exfoliation in aqueous dispersions.

The existence of functional oxygen groups and the degree of oxidation of the different types of carbon nanomaterials was revealed by X-ray photoemission spectroscopy (XPS). Figure 3 shows the corresponding XPS spectra of pCNT, oCNT1, oCNT2 and GO. The survey spectra (Figure 1A) exhibit the $\mathrm{O} 1 \mathrm{~s}$ and the $\mathrm{C} 1 \mathrm{~s}$ core level spectra, which were used to calculate the $\mathrm{C} / \mathrm{O}$ ratio, i.e. the oxidation degree of the respective carbon nanomaterials. Corresponding values are indicated in the figure. The following order of oxidation degree (inverse $\mathrm{C} / \mathrm{O}$ ratio) can be established: pCNT $(1 / 18.05)<$ oCNT2 $(1 / 13.88)<$ oCNT1 $(1 / 8.78)<$ GO $(1 / 4.67)$. While the pristine CNT sample already contains a small amount of oxygen, the oCNT samples exhibit increased oxygen degrees proportional to the applied reflux times of the applied oxidative $\mathrm{HNO}_{3}$ treatment. GO reveals the highest degree of oxidation, thus well reflecting the harsh oxidation conditions of the applied modified Hummer's method. The deconvoluted C1s 
core level spectra (Figure 2B) provide more details on type and relative amount of existing functional oxygen groups.
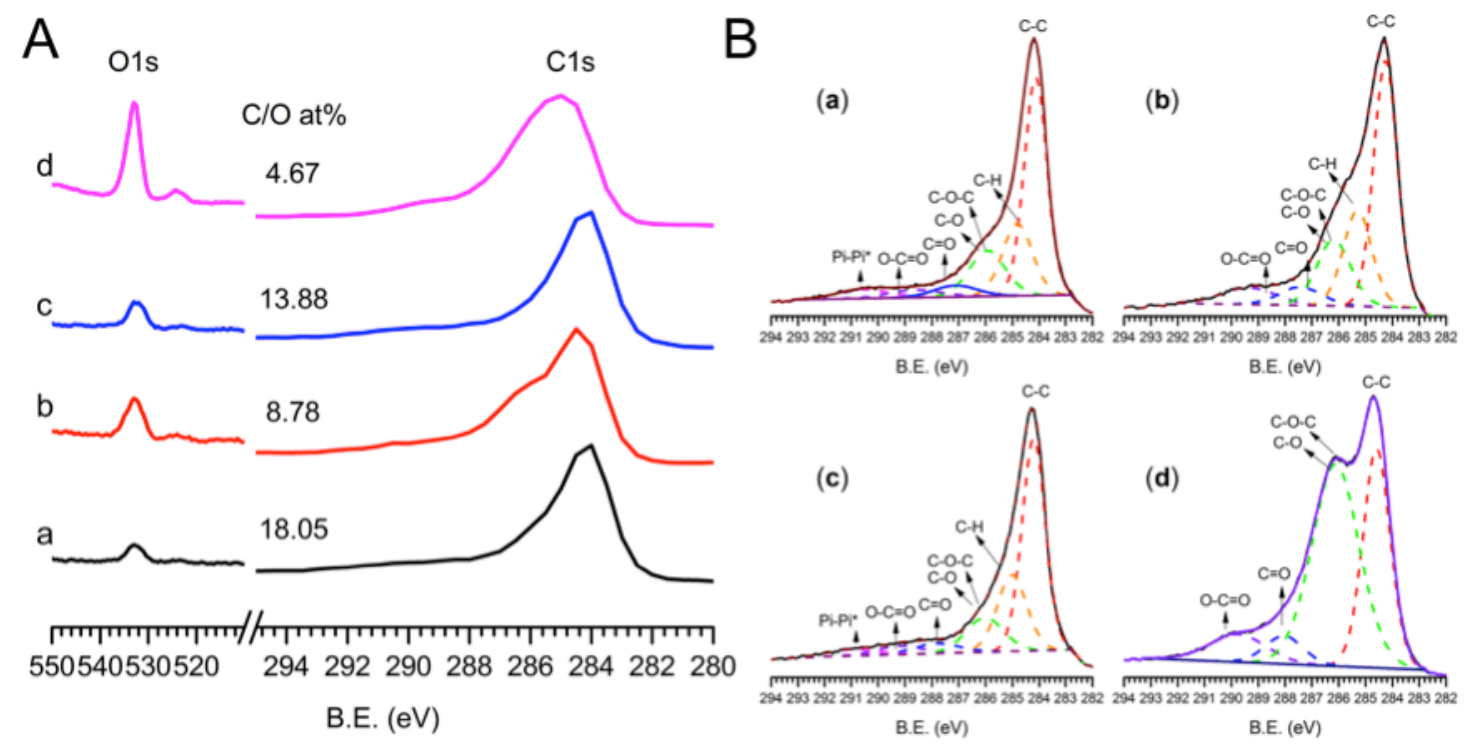

Fig. 3 - XPS spectra of O1s and C1s orbitals of carbon nanostructures and its chemical composition. (A) survey spectra and (B) deconvolution of C1s orbital of (a) pCNT, (b) oCNT1, (c) oCNT2, and (d) GO.

The spectra are dominated by a strong peak at $284.3 \mathrm{eV}$, corresponding to the $\mathrm{sp}^{2} \mathrm{C}-\mathrm{C}$ bond. At higher binding energies the contributions of $\mathrm{C}-\mathrm{H}, \mathrm{C}-\mathrm{O} / \mathrm{C}-\mathrm{O}-\mathrm{C}$ (hydroxyl/ epoxy), $\mathrm{C}=\mathrm{O}$ (carbonyl), $\mathrm{O}-\mathrm{C}=\mathrm{O}$ (carboxyl) bonds and the $\pi-\pi$ transition at $284.9 \mathrm{eV}$, $286.1 \mathrm{eV}, 287.5 \mathrm{eV}, 289.4 \mathrm{eV}$, and $290.6 \mathrm{eV}$, respectively, can be seen [48]. CNT samples consistently reveal the presence of carboxyl, carbonyl, hydroxyl and epoxy groups as main oxygen functionalities [49]. The GO sample shows markedly different features. Here hydroxyl and epoxy groups clearly dominate over carboxyl and carbonyl groups, thus evidencing that the high oxygen degree in GO is largely determined by the oxygen groups located on its basal plane (in agreement with observations from [50, 51]). The observation of clear differences in the oxidation degree of the various carbon nanomaterials as well as the distinctive dominance of basal plane oxygen groups in GO, being also in agreement with results from Raman and TGA studies (see Supporting Data), provides an important base for the understanding of the growth of hydroxyapatite 
and its interaction with the respective nanocarbon support, as discussed in the following sections.

3.2 Hydroxyapatite grown on carbon nanostructures: Morphology, structure, chemical composition, growth and integration

Morphology and structure of hydroxyapatite grown on the various nanocarbon support materials were probed by TEM as shown in Figure 4.
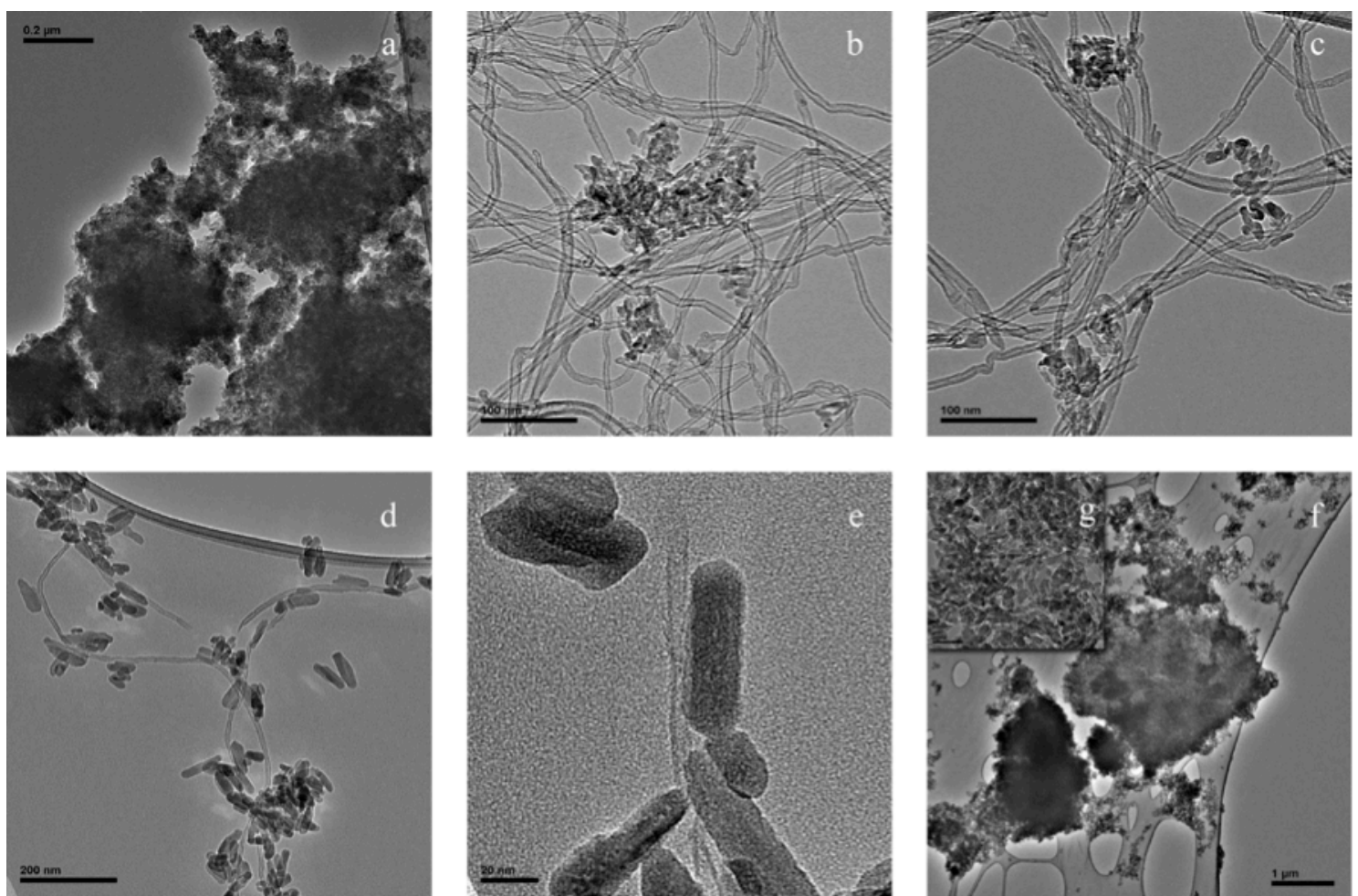

Fig. 4 - TEM micrographs of (a) nHAp, (b) nHAp-pCNT, (c) nHAp-oCNT1, (d, e) nHAp-oCNT2, and (f, g) nHAp-GO.

It can be seen that nHAp itself mainly consists of dense agglomerations of small nanoparticles (Figure 4a). This is a direct consequence of the applied synthesis strategy, which favours a rapid precipitation, nucleation and self-assembly of the precursors into poorly crystallized spherical nHAp nanoparticles, as typically observed in this type of processes [1]. Also in the presence of pCNT and oCNT1 only agglomerates of small nHAp nanoparticles are observed (Figure 4b, c). These are non-uniformly distributed on 
the respective nanocarbon surface. A clearly distinct behaviour is seen for the nHApoCNT2 composite (Figure 4d). Here nHAp show a rod-like structure with lengths between $50 \mathrm{~nm}$ and $80 \mathrm{~nm}$, and cross-sections around $20 \mathrm{~nm}$. The vast majority is directly attached to the oCNT2 support. Please note, that the appearance of isolated nHAp nanorods in Figure $4 \mathrm{~d}$ most likely is an artefact related to the TEM specimen preparation. This requires the application of ultrasonic pulses with high energy densities leading to the eventual detachment of formerly attached nHAp nanorods. At a higher magnification, Figure 4e shows that several nHAp nanorods are laterally aligned along the sidewalls of the oCNT2 support (Figure 4e). The observation that hydroxyapatite forms larger nanorods and that most of them are well attached to the oCNT2 surface, despite the harsh conditions for TEM specimen preparation, points to specific nucleation conditions for hydroxyapatite favouring a preferential growth in the form of nHAp nanorods on the oCNT2 surface accompanied by its strong integration. This is further corroborated by the subsequent XPS and high-resolution TEM studies (see below). In the case of the nHAp-GO composite, also nHAp nanorods are observed (Figure 4f). These are densely distributed on the basal plane of the individual GO sheets reaching a full coverage (Figure $4 \mathrm{~g}$ ). Since the formation of nHAp nanorods implies that nucleation and growth of hydroxyapatite crystals is not random, but occurs along a preferred crystallographic direction, their appearance in nHAp-oCNT2 and nHAp-GO composites suggests that this process is controlled by the presence of functional oxygen groups on the surface of the respective nanocarbon support materials.

Structural information on the nHAp nanocarbon composites is provided by X-ray diffraction (XRD) as shown in Figure 5. The appearance of Bragg reflections at $2 \Theta$ values of $25.95^{\circ}, 28.20^{\circ}, 29.00^{\circ}, 31.85^{\circ}, 32.25^{\circ}, 33.00^{\circ}, 34.15^{\circ}$ and $39.80^{\circ}$ corresponds to the (002), (102), (210), (211), (112), (300), (202) and (130) diffraction planes of 
hydroxyapatite, respectively. The absence of detectable diffraction peaks in the fingerprint region of tricalcium phosphates (TCP) between $30^{\circ}$ and $31^{\circ}$ (as indicated in Figure 3) where the highest diffraction peaks should appear [18], excludes the eventual presence of $\alpha$-TCP and $\beta$-TCP as predominant phases.

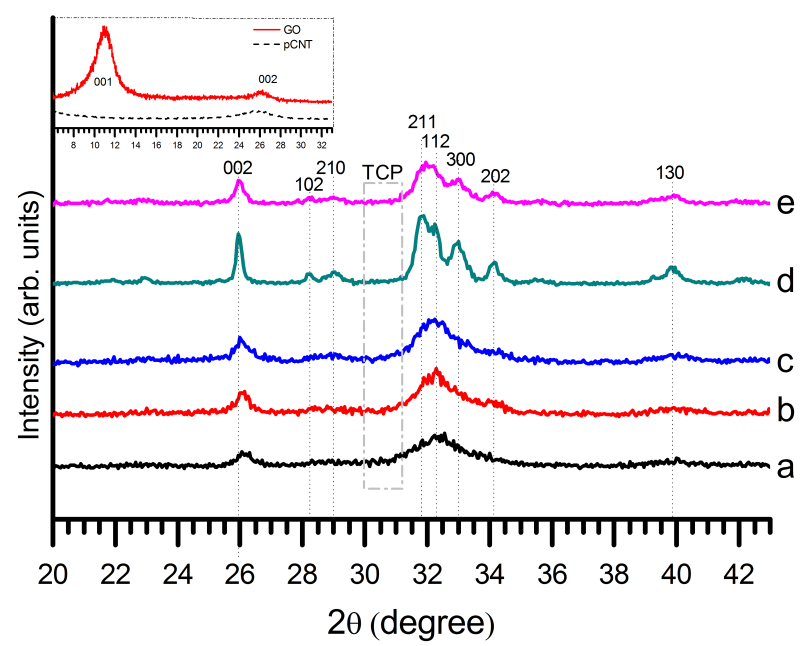

Fig. 5 - XRD patterns of (a) nHAp, (b) nHAp-pCNT, (c) nHAp-oCNT1, (d) nHApoCNT2, and (e) nHAp-GO. Hydroxyapatite planes are indicated as well as the fingerprint region for TCPs. Inset shows the patterns of pCNT and GO.

For the composites nHAp-pCNT, nHAp-oCNT1 the dominant (211), (112), (300), (202) diffraction peak series of hydroxyapatite in the $2 \theta$ range between $30^{\circ}$ and $35^{\circ}$ are rather broad and unresolved, as is the case for the starting nHAp itself. The position of its maximum as well as its broad width is indicative of small crystallite size of hydroxyapatite nanoparticles, as shown in systematic studies on the evolution of the (211) peak with its crystallite size [52]. For this reason, the eventual presence of a minor amorphous calcium phosphate phase cannot be concluded in nHAp, nHAp-pCNT and nHAp-oCNT1 composites. For the composites nHAp-oCNT2 and nHAp-GO, the broad peak between $30^{\circ}$ and $35^{\circ}$ becomes resolved and now shows the prominent hydroxyapatite diffraction features. Most importantly to note is the significant increase of the (300) peak intensity accompanied additionally by an enhancement of the intensity 
of the (002) diffraction peak at $25.95^{\circ}$. The nHAp crystallites in these composites display a pronounced preferred orientation as revealed by the variation of the intensity ratios for the three maximum peaks (211), (300), and (002) [53]. According to ICDD card 72-1243, for a random powder mixture, the reference intensity ratios for (211), (300), (002) are $\mathrm{RI}_{(211)} / \mathrm{RI}_{(300)}=1.82$ and $\mathrm{RI}_{(211)} / \mathrm{RI}_{(002)}=2.38$. Divided by the corresponding intensity ratios of the measured peaks the degree of texture index $R_{h k l}=$ $\left(\mathrm{I}_{(211)} / \mathrm{I}_{(\mathrm{hkl})}\right) /\left(\mathrm{RI}_{(211)} / \mathrm{RI}_{(\mathrm{hkl})}\right)$ is calculated (Table 1). Values lower than 1.0 describe a preferred growth orientation, whereas the (300) direction is associated with a rod-like growth and the (002) direction with a plate-like growth of hydroxyapatite [54]. Preferential growth in both directions is clearly observed for the nHAp-oCNT2 and nHAp-GO composites. This also relates to the calculated mean hydroxyapatite crystallite sizes (Table 1). The values for composites nHAp-oCNT2 and nHAp-GO are with $30 \mathrm{~nm}$ and $18 \mathrm{~nm}$, respectively, by far the largest of all samples. Composites nHAp-oCNT1 and nHAp-pCNT exhibit a significantly lower crystal size of $11 \mathrm{~nm}$. Remarkably, with a value of only $8 \mathrm{~nm}$, nHAp itself reveals the lowest crystal size. These observations also correlate well with the TEM results from Figure 4. Finally, it remains to comment that the XRD peaks of the hydroxyapatite materials are not influenced by those of CNTs or GO, as shown in the inset of Figure 5. The pattern for pCNT is characterized by a broad diffraction peak at about $26^{\circ}$ corresponding to the (002) planes formed by the multi-walled layers of pCNT (Please note that oxidized CNTs show the same pattern since the presence of oxygen functionalities on the outer walls does not affect the (002) spacing in agreement with the TEM observations). Its intensity compared to the rather sharp (002) hydroxyapatite peak is negligible. On the other hand, GO is dominated by a strong peak at $11^{\circ}$, which is outside the region characteristic for hydroxyapatite. This peak is typical for GO comprised of a few layers 
with an enhanced interlayer spacing of $0.8 \mathrm{~nm}$ due to the presence of basal plane oxygen functionalities (epoxies and hydroxyl groups) and residual water [55]. Additionally, a low intensity peak at $26^{\circ}$ indicates minor traces of remaining nonexfoliated graphitic material.

Table 1. Texture index, crystallite size (calculated from XRD) and $\mathrm{Ca} / \mathrm{P}$ ratio (calculated from XPS)

\begin{tabular}{lcccc}
\hline Samples & $\begin{array}{c}\text { Texture } \\
\mathbf{R}_{(\mathbf{3 0 0})}\end{array}$ & $\begin{array}{c}\text { Texture } \\
\mathbf{R}_{(\mathbf{0 0 2})}\end{array}$ & $\begin{array}{c}\text { Crystallite size } \\
\mathbf{X}_{\mathbf{s}}(\mathbf{n m})\end{array}$ & $\mathbf{C a} / \mathbf{P}$ \\
\hline nHAp & 1.08 & 0.84 & 8 & 1.55 \\
nHAp-pCNT & 1.12 & 0.79 & 11 & 1.51 \\
nHAp-oCNT1 & 0.90 & 0.67 & 11 & 1.56 \\
nHAp-oCNT2 & 0.85 & 0.57 & 30 & 1.56 \\
nHAp-GO & 0.87 & 0.70 & 18 & 1.41 \\
\hline
\end{tabular}

More details concerning crystal structure and growth of nHAp on carbon surfaces is obtained by high resolution TEM (HRTEM) analyses as shown in Figure 6. Figure 6a presents a TEM image of a nHAp nanorod laterally attached to the CNT surface as representative case in the nHAp-oCNT2 composite. A HRTEM image (Figure 6b) of the nHAp-oCNT2 interaction zone clearly reveals the high crystalline structure of the nHAp nanorod and also shows the walls of the carbon nanotube. A fast Fourier transform (FFT) diffractogram of the indicated zone (Figure 6b) shows the well-defined spots for the (002) and (300) planes of hydroxyapatite. These can be clearly identified in the corresponding HRTEM image shown in the inset of Figure 6c). Apparently, the (300) planes of nHAp nanorods enable interactions with the carbon surface and define an oriented growth of hydroxyapatite in its crystallographic a-direction along the sidewalls of carbon nanotubes, as seen for the case of the nHAp-oCNT2 composite. In the case of the nHAp-GO composite the interaction with the (300) planes of hydroxyapatite takes place on the basal plane of GO. Due to its planar morphology the 
crystallographic a-direction of hydroxyapatite can adapt any orientation along the carbon surface enabling the growth of nHAp nanorods in random directions. While in this way a dense coverage with nHAp nanorods can be obtained (see Figure 4), simultaneous growth of nHAp nanorods in different directions induces steric limitations on the continuous growth. This yields in a somewhat smaller crystallite size of the nHAp nanorods in the nHAp-GO composite compared to the one in the nHAp-oCNT2 composites, as revealed by XRD results (Table 1).

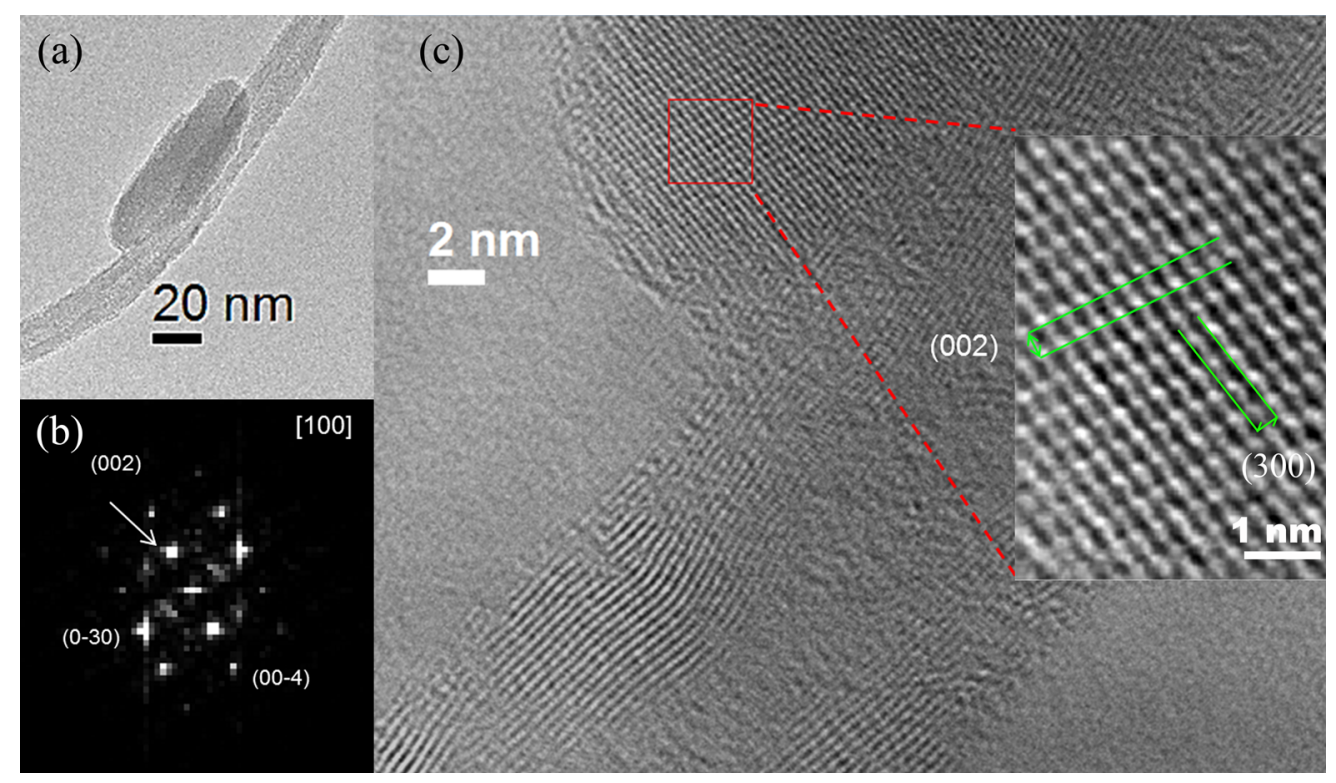

Fig. 6 - (a-c) HRTEM images and FFT diffractogram of a nHAp nanorod grown on oCNT2.

Interactions of the (300) plane of hydroxyapatite with the carbon surface were also proposed for the cases of carbon nanotubes [18] and reduced graphene oxide [37]. The corresponding models are based on purely geometric considerations trying to establish a lattice match of calcium atoms of the (300) plane with carbon atoms arranged in a perfect hexagonal network not taking into account chemical interaction possibilities except van der Waals interactions. However, by applying wet-chemical processes, it is well known that the growth of hydroxyapatite on a surface containing oxygen 
functionalities is established by chemical coordination interactions between precipitating $\mathrm{Ca}^{2+}$ ions and oxygen groups $[30,31,34,36,40,41]$.

A closer look at the crystal structure of hydroxyapatite helps to better understand the issues of interaction and preferred growth into the form of nanorods on carbon surfaces containing functional groups. Hydroxyapatite crystallizes in a hexagonal structure and is part of the $\mathrm{P}_{3} / \mathrm{m}$ space group, characterized by a sixfold $\mathrm{c}$-axis perpendicular to equivalent a and b-axes at $120^{\circ}$ angles to each other [56, 57]. Phosphate anions form the skeleton of the unit cell arranged in two channels A and B along the c axis. In the two channels A the oxygen atoms from the phosphate groups and the calcium ions of type II, denominated $\mathrm{Ca}(\mathrm{II})$, are found at heights of $1 / 4$ and $3 / 4$, respectively. The two channels B contain calcium ions of type $\mathrm{I}$, denominated $\mathrm{Ca}(\mathrm{I})$, each at heights 0 and $1 / 2$. An atomic model of the unit cell of hydroxyapatite is presented in Figure 7. The positions of the $\mathrm{Ca}, \mathrm{P}, \mathrm{O}$ atoms and $\mathrm{OH}$ groups are indicated, and the planes (300) and (002) are included. Views along the a-direction (left) and in the a-b plane (right) are offered. As can be seen, the (300) plane hosts a $\mathrm{Ca}(\mathrm{I})$ atom located at the intersection with the (002) plane at height zero. This $\mathrm{Ca}(\mathrm{I})$ atom coordinates with six oxygen atoms belonging to the next unit cell. In other words, when the (300) plane is in contact with an oxygenated carbon surface, the $\mathrm{Ca}(\mathrm{I})$ atom easily can coordinate with up to six oxygen atoms from functional groups at the external surface thus enabling $\mathrm{O}-\mathrm{Ca}(\mathrm{I})-\mathrm{O}$ interactions of ionic and even covalent nature. In the case of carbon nanotubes these interactions are established with oxygen atoms principally originating from carboxyl and carbonyl groups located at defects sites of their sidewalls. In the case of GO hydroxyl and epoxy groups at the basal plane dominate as interaction sites. A generic structure for an oxidized nanocarbon surface containing the different types of functional oxygen groups available on the sidewall and basal plane of carbon nanotubes and GO, 
respectively, is depicted below the hydroxyapatite unit cell drawn along its a-direction in order to indicate the various interaction possibilities with the $\mathrm{Ca}(\mathrm{I})$ atom of the $(300)$ plane.

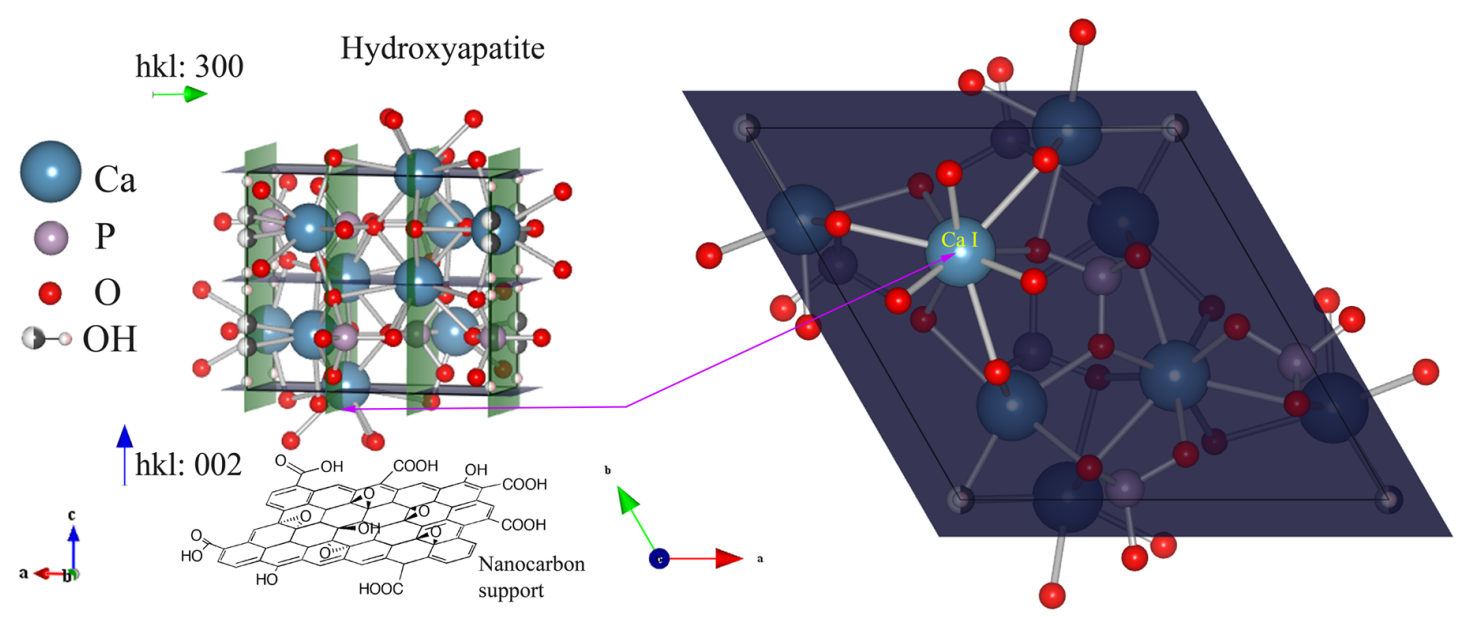

Fig. 7 - Atomic structure representation of the unit cell of hydroxyapatite indicating the positions of the $\mathrm{Ca}, \mathrm{P}, \mathrm{O}$ atoms and $\mathrm{OH}$ groups, as well as the (300) and (002) planes in green and blue color, respectively. The left structure is oriented along the a-direction. A generic model for an oxidized nanocarbon surface containing different types of oxygen groups is placed below the (300) planes of the unit cell of hydroxyapatite indicating interaction possibilities between different types of oxygen groups and the $\mathrm{Ca}(\mathrm{I})$ atom in the (300) plane. The right structure depicts the projection of the unit cell of hydroxyapatite in the a-b plane showing the $\mathrm{Ca}(\mathrm{I})$ atom with its interaction possibilities outside the unit cell.

The distance between two equivalent next neighbour $\mathrm{Ca}(\mathrm{I})$ atoms located at (300) planes able to interact with external oxygen functionalities is $0.81 \mathrm{~nm}$. An adequate density of functional oxygen groups at the surface of nanocarbon materials might match with this distance and enable a preferential growth in the form of nanorods along the carbon surface as observed in the case of oCNT2 and GO. However, considering the irregular distribution of different types of oxygen functionalities on the carbon surface there seems to be certain flexibility in what concerns the coordination of $\mathrm{Ca}(\mathrm{I})$ atoms with oxygen groups from the carbon surface. The coordination number may vary and 
the interaction does not have to be orthogonal. The positions of the atoms within the hydroxyapatite lattice thus adapt to the conditions of the support. Mechanical flexibility of the carbon support may further contribute to establish improved coordination between $\mathrm{Ca}$ and oxygen groups on the carbon surface. Thus, favourable conditions are created for enabling nucleation and growth of nHAp nanorods along the oxidized sidewalls and basal planes for carbon nanotubes and GO, respectively. The model proposed not only explains the rod-like growth of hydroxyapatite but also the strong integration onto the support due to the formation of favourable O-Ca-O coordination interaction.

More details on the chemical composition and the issue of chemical integration of hydroxyapatite on the different types of oxidized carbon supports are obtained by X-ray photoelectron spectroscopy (XPS). Figure 8 shows the XPS C1s, O1s, Ca2p and P2p core level spectra of nHAp and the respective composite materials. From the integrated area of the $\mathrm{Ca} 2 \mathrm{p}$ and $\mathrm{P} 2 \mathrm{p}$ peaks information about the phase purity of hydroxyapatite is obtained by calculating the $\mathrm{Ca} / \mathrm{P}$ ratio. This affords $\mathrm{Ca} / \mathrm{P}$ values of $1.55,1.51,1.56,1.56$ for nHAp, nHAp-pCNT, nHAp-oCNT1 and nHAp-CNT2, respectively (see Table 1). These ratios match well with those found for natural bone which, depending on its type and age, range from 1.5 to 1.67 [3]. This suggests that the taken synthesis route in all cases favours the formation of non-stoichiometric calcium deficient hydroxyapatite, i.e. $\mathrm{Ca}_{10}\left(\mathrm{PO}_{4}\right)_{w}\left(\mathrm{HPO}_{4}\right)_{x}\left(\mathrm{CO}_{3}\right)_{y}(\mathrm{OH})_{z}$, with a relative low content of $\mathrm{CO}_{3}{ }^{2-}$ and $\mathrm{HPO}_{4}^{2-}$ impurities. On the other hand, the nHAp-GO composite with a value of 1.41 shows the lowest $\mathrm{Ca} / \mathrm{P}$ ratio and points to the existence of higher amounts of impurities inserted in the nHAp lattice. This induces lattice strain [58] and results in a crystallite size somewhat smaller than for the case of nHAp-oCNTs (see table 1) pointing to a fast nucleation process due to the high amount of accessible oxygen functionalities in GO. 


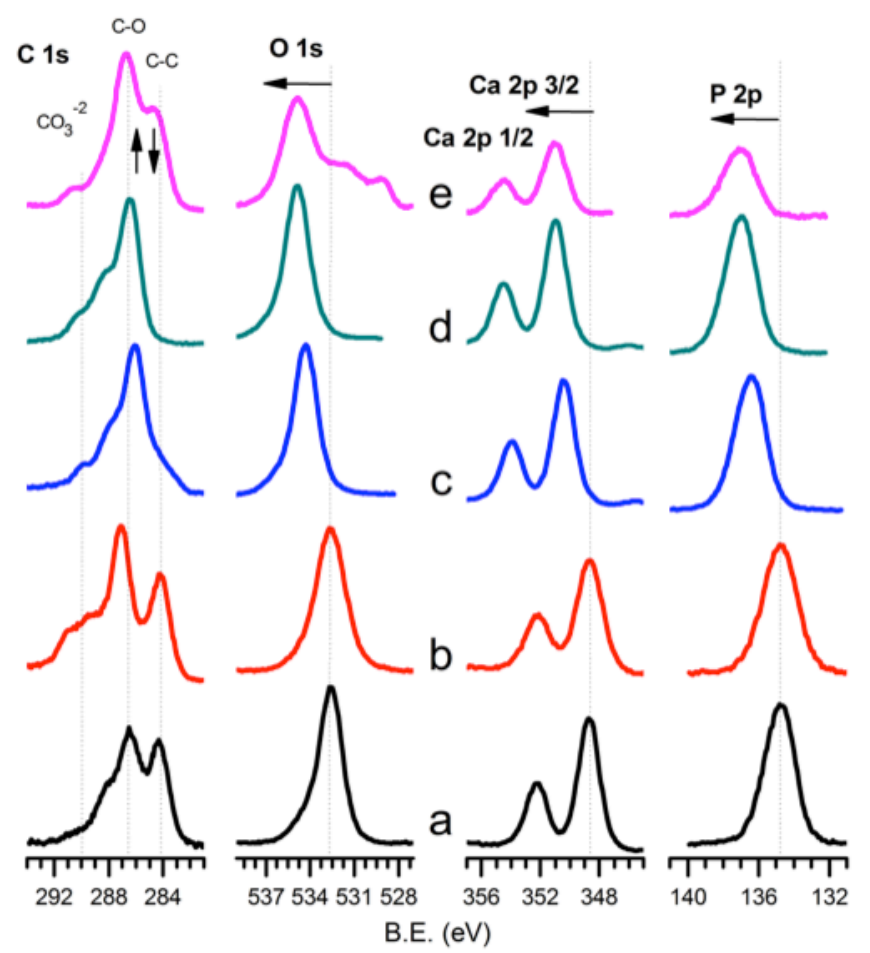

Fig. 8 - XPS spectra of C1s, O1s, Ca2p and P2p orbitals of (a) nHAp, (b) nHAppCNT, (c) nHAp-oCNT1, (d) nHAp-oCNT2, and (e) nHAp-GO. Lines indicate the reference position of the elements in nHAp.

By making use of the well-known fact that shifts in the positions of XPS binding energies reflect changes in the chemical environment of the probed elements $[59,60]$ important insights on the interface interactions between nHAp and the carbon support can be obtained thus revealing the degree of chemical integration. (Please note that the term chemical integration should not be confused here with the issue of mechanical integration. This relationship is not straightforward and is subject to proper mechanical testing). While the $\mathrm{C} 1 \mathrm{~s}, \mathrm{O} 1 \mathrm{~s}, \mathrm{Ca} 2 \mathrm{p}$ and $\mathrm{P} 2 \mathrm{p}$ core level spectra for the different materials present almost identical features important changes are noted concerning their binding energies. The values for the $\mathrm{O} 1 \mathrm{~s}, \mathrm{Ca} 2 \mathrm{p}_{1 / 2}, \mathrm{Ca} 2 \mathrm{p}_{3 / 2}, \mathrm{P} 2 \mathrm{p}$ peaks for nHAp are located at $532.6 \mathrm{eV}, 352.2 \mathrm{eV}, 348.7 \mathrm{eV}$ and $134.8 \mathrm{eV}$, respectively, and are taken as reference values. The origin for the presence of $\mathrm{C} 1 \mathrm{~s}$ peaks in nHAp is not clear, but widely recognized in hydroxyapatite samples $[58,59,61]$. Its presence is beneficial for the 
charge correction of the XPS spectra. No changes in binding energies are observed in the nHAp-pCNT sample and thus reveal the lack of chemical interactions between the agglomerated hydroxyapatite nanoparticles and the nanotube surface. Therefore, this sample is rather a mixture than a composite material. This situation changes as soon as oxygen functionalities are present on the nanocarbon support materials. The nHApoCNT1 material shows systematic shifts of the (O1s, Ca2p-3/2 and P2p) peak positions towards higher binding energies by $(1.8 \mathrm{eV}, 1.8 \mathrm{eV}$, and $1.6 \mathrm{eV})$. The up-shifts are even more pronounced for the nHAp-oCNT2 material $(2.2 \mathrm{eV}, 2.3 \mathrm{eV}, 2.2 \mathrm{eV})$ and even higher for the nHAp-GO material $(2.2 \mathrm{eV}, 2.4 \mathrm{eV}, 2.3 \mathrm{eV})$. No changes in the $\mathrm{C} 1 \mathrm{~s}$ peak positions are observed. These observations confirm the existence of significant chemical interactions between the grown nHAp and the oxidized nanocarbon support material. Here genuine composite materials are formed. It further becomes clear that the interactions are related to the presence of oxygen groups. Moreover, systematically enhanced binding energies for both, calcium and oxygen atoms underline that the interactions take place between these two elements, thus corroborating the former HRTEM and modelling results suggesting favourable $\mathrm{O}-\mathrm{Ca}(\mathrm{I})-\mathrm{O}$ coordination possibilities. Related up-shifts of binding energies in the P2p spectra most likely reflect an adjustment in bonding distances within the whole nHAp lattice as a direct consequence of enhanced $\mathrm{O}-\mathrm{Ca}(\mathrm{I})-\mathrm{O}$ coordination possibilities. Finally, the fact that highest chemical interactions are observed for the nHAp-oCNT2 and nHAp-GO composites where nHAps exhibit a rod-like structures of high crystallite sizes (see table 1) underlines the close relation between $\mathrm{O}-\mathrm{Ca}(\mathrm{I})-\mathrm{O}$ interactions, chemical integration on the carbon support, and preferential growth of hydroxyapatite along its crystallographic a-direction. Taking together all these results the growth of nHAp on the carbon surfaces with different amounts of functional oxygen groups, as depicted in Figure 9, can be 
described as follows: (a) The absence of oxygen functionalities on carbon nanotubes leads to the formation of spherical nHAp or agglomerates thereof not producing any interaction (case pCNT). (b) A high amount of oxygen functionalities on carbon nanotubes produces many competing nucleation points for $\mathrm{Ca}^{2+}$ precipitation. Being distributed on a relatively small and curved cylindrical carbon surface induces steric limitations and thus frustrates an undisturbed simultaneous growth of neighbouring nHAp cyrstallites along the preferred crystallographic growth direction into rod-like nHAp. Spherical nHAp or agglomerates thereof are formed. However, in contrast to the former case, these are partly integrated on the CNT surface (case oCNT1). (c) An amount of oxygen functionalities on carbon nanotubes in between the former two cases provides an adequate amount of nucleation points for $\mathrm{Ca}^{2+}$ precipitation enabling an undisturbed growth of nHAp on the cylindrical CNT surface into highly crystalline rodlike structures exhibiting an enhanced degree of integration (case CNT2). (d) On the other hand, dimension and morphology of the nanocarbon surface have to be taken into account as well, as shows the case of GO. For all the nanocarbon support materials probed, GO is the one with the highest amount of oxygen functionalities available as nucleation site for precipitating $\mathrm{Ca}^{2+}$. In contrast to a highly oxidized and small cylindrical CNT surface, the rather planar and large surface of GO offers an additional degree of freedom by enabling rod-like growth in random orientations. In this way nHAp nanorods easily can reach an almost full coverage of a graphene oxide as well as a very high integration degree. All these examples illustrate that length, crystallite size, and integration degree of nHAp nanorods are intimately linked to each other and largely depend on the amount of functional oxygen groups on a given nanocarbon surface and its availability for precipitating $\mathrm{Ca}^{2+}$ ions. The comparison between CNTs and GO 
reveals that morphology and dimension of the nanocarbon surface itself are additional factors of influence.

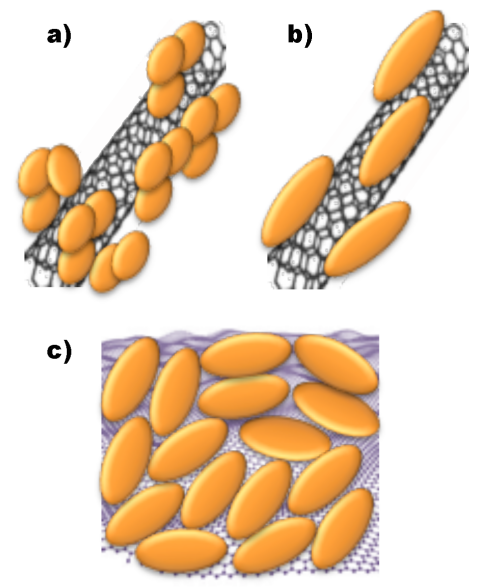

Fig. 9 - Scheme illustrating the growth of nHAp on nanocarbon surfaces with different different oxidation degree. (a) oCNT1, (b) oCNT2, and (c) GO.

\subsection{In vitro bioactivity behaviour of $n H A p$-nanocarbon composites}

In-vitro bioactivity tests were carried out using body fluid (SBF) as a standard precursor solution mimicking the mineralization process of bone. In order to evaluate the biomineralization ability of the nHAp-nanocarbon composites expressed by the formation of apatite on the respective surfaces the composite materials were exposed to SBF for times of one and four weeks. Corresponding test on control samples not containing nHAp, i.e. pCNT, oCNT2, as well as graphene oxide and reduced graphene oxide did not provide any evidence of apatite formation (see Suppl. Data). This is well in agreement with results reported in literature [38, 41]. On the contrary, all types of nHAp-nanocarbon composites reveal the formation of apatite upon bioactivity tests as consistently confirmed by XRD, SEM-EDX and XPS results (see Suppl. Data). These observations clearly demonstrate that the presence of hydroxyapatite is required to 
establish a bioinspired surface that can trigger the self-assembly of apatite. TEM images (Figure 10) illustrate the bioactivity behaviour of the various nHAp-nanocarbon composites after SBF immersion times of one and four weeks.
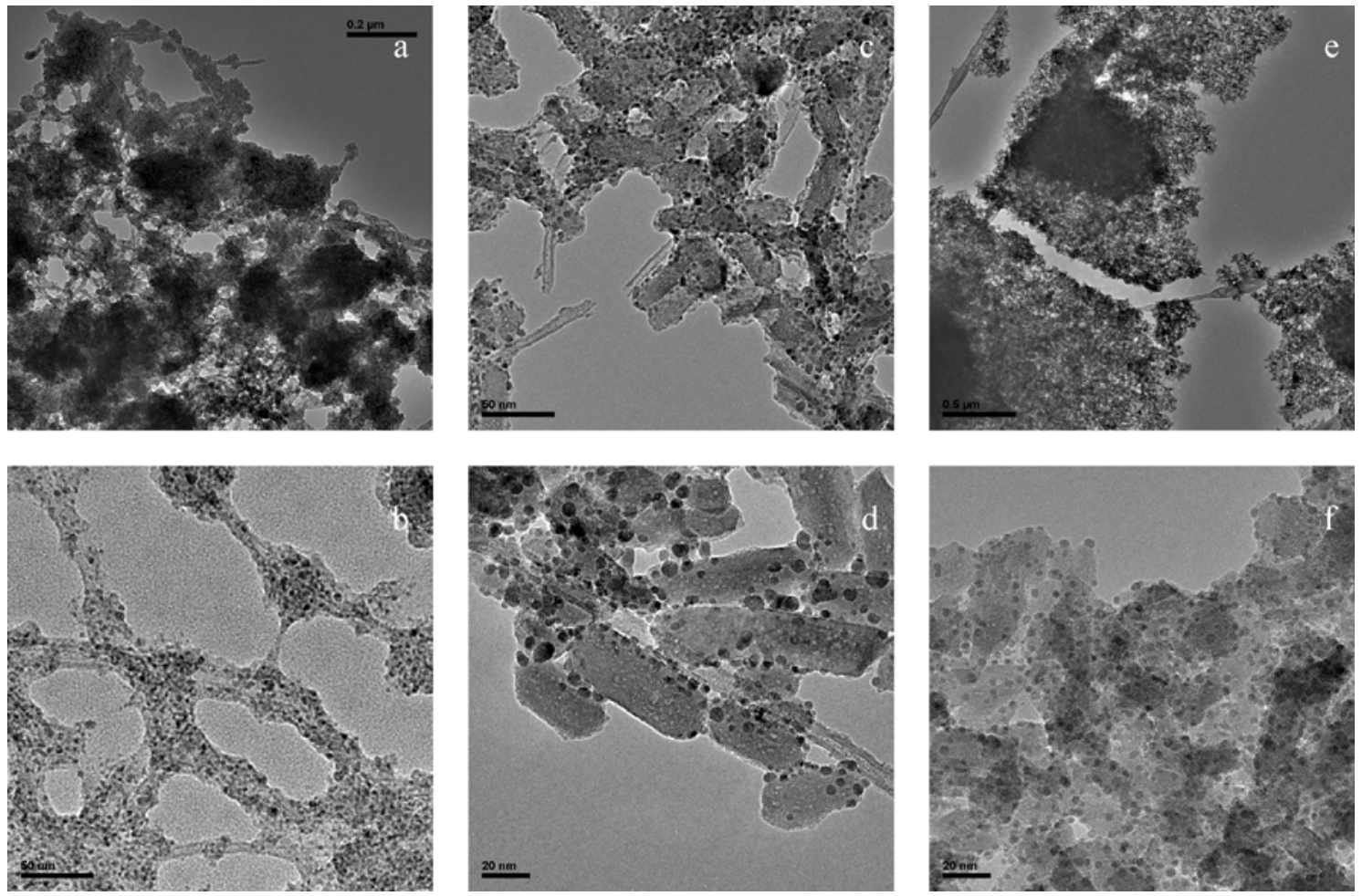

Fig. 10 - TEM micrographs of (a, b) nHAp-oCNT1, (c, d) nHAp-oCNT2, and (e, f) nHAp-GO after 1 week (upper row) and 4 weeks (lower row) of bioactivity

The evolution of the surface of nHAp-oCNT1 composite after 1 and 4 weeks of time (Figure $10 \mathrm{a}, \mathrm{b}$ ) resembles a biomineralization process characterized by an induction period. During the first week a non-continuous discrete coating is has been formed. Available oxygen functionalities on oCNT1 and nHAp both act as competing nucleation points for ionic cluster precursors not yet forming apatite nanoparticles. Only after four weeks of bioactivity these precursors are transformed into small apatite nanoparticles (nAps) on the composite surface. Their formation is induced by the presence of nHAp which allows for selective ion diffusion towards the formation of apatite, a situation similar to what is observed for sintered hydroxyapatite exposed to SBF [62]. In the case of nHAp-oCNT2 the formation of apatite nanoparticles is already seen after the first 
week of bioactivity (Figure 10c). They specifically grow on the nHAp nanorods, and interestingly, few of them appear in-between the interface of nHAp nanorods and the oCNT2 surface. This observation suggests that nAp nuclei forming on the nHAp surface retain a high reactivity which allows them to migrate towards the nHAp-oCNT2 interface to initiate nAp growth also at this site, thus consequently leading to the disruption of the established integration as will be shown by the subsequent XPS studies. After four week of bioactivity no further changes on the growth of nAp nanoparticles are observed. Likewise, the nHAp-GO composite reveals nucleation and growth of nAp nanoparticles on the surface of the nHAp nanorods after the first week of bioactivity (Figure 10e). However, nAp nanoparticles are not found in-between the nHAp-GO interface. Here the dense coating of the GO surface by the nHAp nanorods significantly reduces the accessibility of migrating nAp nuclei to the reach the respective nHAp-GO interface. Figure 11 provides a graphical illustration of the bioactivity on the three types of nHAp-nanocarbon composites.

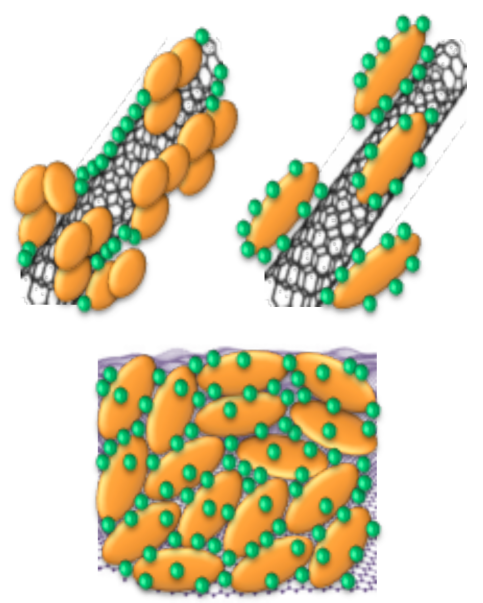

Fig. 11 - Graphical illustration of the formation of apatite nanoparticles on the surfaces of the nHAp-nanocarbon composites. (a) nHAp-oCNT1, (b) nHApoCNT2, and (c) nHAp-GO (top-view). 
The formation of apatite nanoparticles on the various composites surfaces is followed more closely by XPS spectroscopy (Figure 12). The O1s, Ca2p, and P2p core-level spectra reveal systematic changes in the respective binding energies of the $\mathrm{O} 1 \mathrm{~s}, \mathrm{Ca} 2 \mathrm{p}$ and $\mathrm{P} 2 \mathrm{p}$ peaks at different stages of the bioactivity process (Figure 10).

(b)

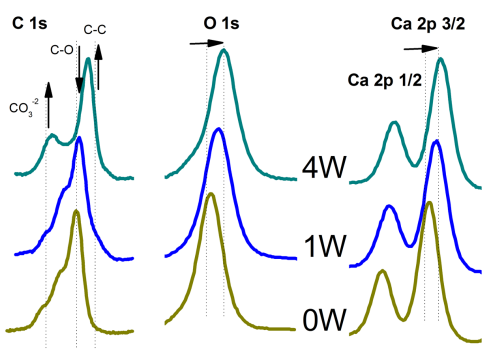

(a)

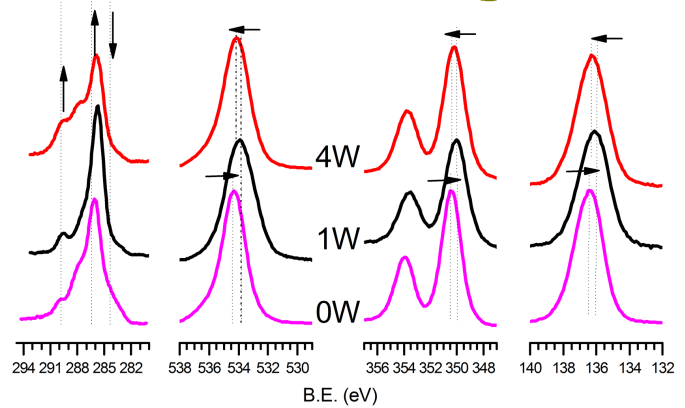

(c)
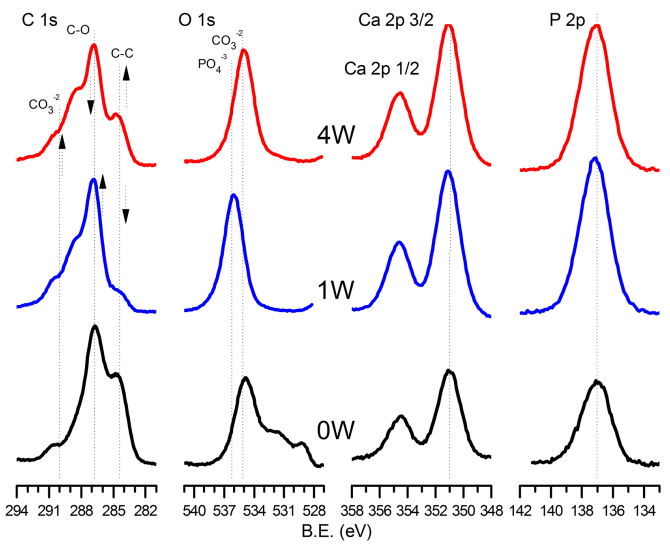

Fig. 12 - XPS spectra of C1s, O1s, Ca2p, and P2p orbitals of (a) nHAp-oCNT1, (b) nHAp-oCNT2 (c) nHAp-GO before (0w), after 1 week (1w), and 4 weeks (4w) of bioactivity, respectively.

For nHAp-oCNT1, after week 1, the binding energies of the XPS peaks experience discrete down-shifts of $-0.4 \mathrm{eV}(\mathrm{O} 1 \mathrm{~s}),-0.4 \mathrm{eV}(\mathrm{Ca} 2 \mathrm{p})$, and $-0.3 \mathrm{eV}(\mathrm{P} 2 \mathrm{p})$, as shown in Figure 12a. However, after week 4, this trend is reversed by slight up-shifts of $0.2 \mathrm{eV}$ for all the respective peaks. Thus a weakening of the original degree of integration between nHAp and oCNT1 is taking place in the induction period. Only ones apatite has been formed, the original situation is established. For nHAp-oCNT2, after week 1, pronounced down-shifts in the binding energies of $-0.5 \mathrm{eV}(\mathrm{O} 1 \mathrm{~s}),-0.6 \mathrm{eV}(\mathrm{Ca} 2 \mathrm{p})$, and $0.5 \mathrm{eV}(\mathrm{P} 2 \mathrm{p})$ are observed (Figure 12b). After week 4, further lowering of the binding energies by $-0.3 \mathrm{eV}(\mathrm{O} 1 \mathrm{~s}),-0.4 \mathrm{eV}(\mathrm{Ca} 2 \mathrm{p})$, and $-0.5 \mathrm{eV}(\mathrm{P} 2 \mathrm{p})$ is noted. These changes in binding energies clearly show that nAp nanoparticles growing in-between the nHAp- 
oCNT2 interface contribute to a significant weakening original interface interactions upon the process of bioactivity. In the case of nHAp-GO (Figure 12c) no changes in the peak positions of the $\mathrm{Ca} 2 \mathrm{p}$ and $\mathrm{P} 2 \mathrm{p}$ core level spectra can be seen. Obviously, the formation of $\mathrm{nAp}$ nanoparticles on the nHAp surface does preserve the original integration degree, in agreement with the observation that no nAp nanoparticles are formed in-between the nHAp-GO interface. Therefore this composite is characterized by a high integration stability throughout the process of bioactivity. Remarkably, this is accompanied by a significant change in the O1s spectra. After 1 week, the main O peak shifts to higher binding energies by $1.2 \mathrm{eV}$ and, after 4 weeks, a down-shift by $-1.1 \mathrm{eV}$, is taking place, almost restoring the original value of nHAp-GO. These type of changes are indicative for remineralization processes based on the exchange between $\mathrm{PO}_{4}{ }^{3-}$ ions and $\mathrm{CO}_{3}{ }^{2-}$ ions in the nHAp lattice. After the first week of bioactivity, nAP formation on nHAp is characterized by a relative up-take of $\mathrm{PO}_{4}{ }^{3-}$ substituting $\mathrm{CO}_{3}{ }^{2-}$ impurities in the nHAp lattice. This remineralization prompts a displacement of the O1s peak towards higher binding energies. On the contrary, after 4 week of bioactivity, a reversed ionexchange process is taking place. Now $\mathrm{CO}_{3}{ }^{2-}$ is inserted in the nHAp lattice on cost of $\mathrm{PO}_{4}^{3-}$ lattice ions, thus restoring the original integration composition of the degree of the nHAP-GO composite.

The changes in the integration degree of the different nHAp-nanocarbon composites during the bioactivity process are accompanied by modifications in their overall chemical composition, their $\mathrm{Ca} / \mathrm{P}$ ratio and their crystallinity. Corresponding data were obtained from the analyses of the XPS survey spectra and the XRD diffractograms (see Supporting Data). The overall evolution is resumed in Figure 13, which provides valuable insights on the bioactivity mechanism of the composites. 
(a)

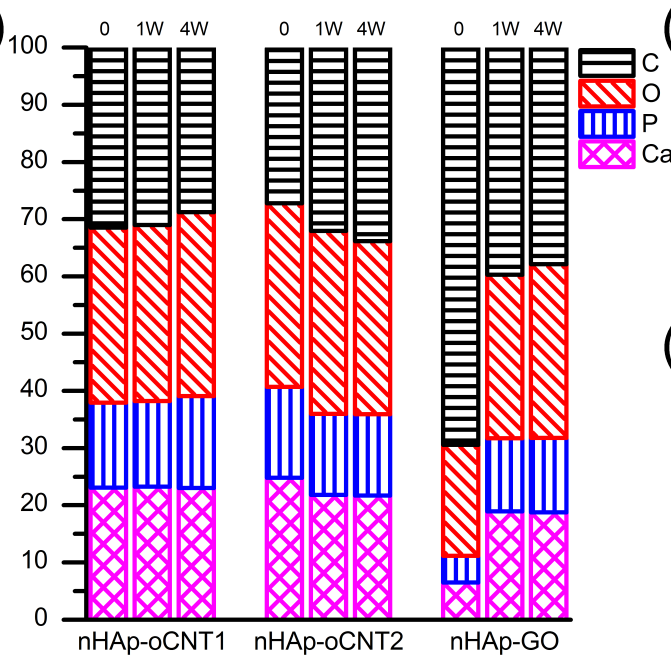

(b)

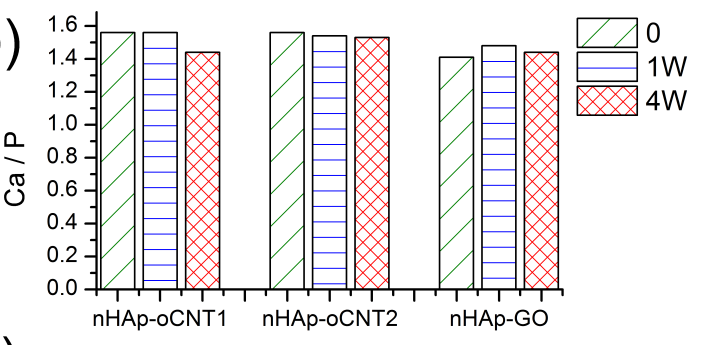

(c)

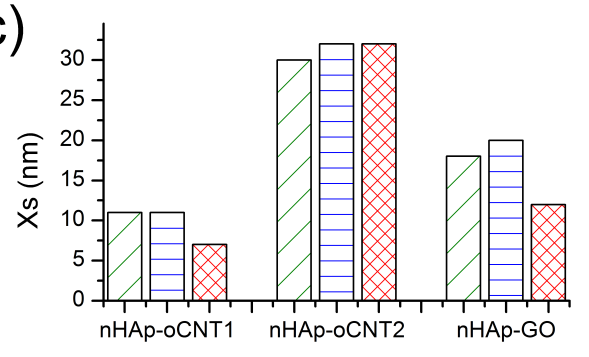

Fig. 13 - Evolution of nHAp and nHAp-CNT/GO composites upon bioactivity assays. (a) Elemental composition from XPS analyses, (b) $\mathrm{Ca} / \mathrm{P}$ ratio from XPs analyses, and c) crystalline size $X_{s}$ from XRD analyses.

For composite nHAp-oCNT1 significant changes only are only perceived after 4 weeks of bioactivity (Figures 13). This correlates with the existence of an induction period for the formation of small nAp nanoparticles. These grow in addition to the existing nHAp nanoparticles supported on the CNT1 surface and contribute to a decrease of the average crystallite size of the overall apatite $(\mathrm{nHAp}+\mathrm{nAp})$ system from the initial $11 \mathrm{~nm}$ to $7 \mathrm{~nm}$ (Figure 13c). This is accompanied by a lower $\mathrm{Ca} / \mathrm{P}$ ratio, which dropped from the initial value of 1.56 to 1.44 , probably due to the inclusion of carbonate defects in the grown nAp nanoparticles. These cause stress in the apatite lattice and lead to a decrease of its average crystallite size. Evidence of inclusions of carbonate defects can be found in the XPS spectra in Figure 11a where the C1s core level spectra shows enhanced intensities for the signal assigned to $\mathrm{CO}_{3}{ }^{2-}$ after 4 weeks of bioactivity. In the case of composite nHAp-oCNT2 relevant changes are perceived after the first week of bioactivity (Figure 13). The bioactivity mechanism for the nHAp-oCNT2 composite is described by the growth of nAp nanoparticles on the surface of nHAp, while simultaneous remineralization processes take place through ion exchange of $\mathrm{CO}_{3}^{2-}$ and 
$\mathrm{PO}_{4}^{3-}$, thus releasing some stress from the nHAp lattice. This argumentation is supported by the decrease of the $\mathrm{Ca} / \mathrm{P}$ ratio from 1.56 to 1.53 (Figure $13 \mathrm{~b}$ ) and a slight increase of crystallite size from $30 \mathrm{~nm}$ to $32 \mathrm{~nm}$ (Figure 13c) of the overall apatite (nHAp+nAp) system. However, inclusion of carbonate defects into the nAp nanoparticles should be also considered, since the intensity of the $\mathrm{CO}_{3}{ }^{2-}$ signal in the $\mathrm{C} 1 \mathrm{~s}$ spectra increased after week 1 and 4 (Figure 13b). For the bioactivity mechanism of the composite nHAp-GO two different transient stages can be encountered: Important changes are observed after the first week of bioactivity (Figure 13). The high increase of the amount of Ca (Figure 13a) and the $\mathrm{Ca} / \mathrm{P}$ ratio (Figure $13 \mathrm{~b}$ ) from 1.41 to 1.48 indicate that a fast nucleation of apatite's precursors was induced by the compact layer of nHAp formed on GO. Similarly to nHAp-oCNT2, apatite deposition on nHAp induces a remineralization process through ion exchange of $\mathrm{CO}_{3}{ }^{2-}$ by $\mathrm{PO}_{4}^{3-}$ accompanied by a slight increase in crystallite size from $18 \mathrm{~nm}$ to $20 \mathrm{~nm}$. In the second stage of bioactivity, the content of $\mathrm{Ca}$ remains almost constant while the $\mathrm{Ca} / \mathrm{P}$ ratio decreases to 1.44 . Thus, this process is governed by the formation of small nAp nanoparticles incorporating $\mathrm{CO}_{3}{ }^{2-}$ defects, being consistent with a significant reduction of average crystallite size of the overall apatite (nHAp+nAp) system to $12 \mathrm{~nm}$ (Figure 13c).

These results clearly show that the bioactivity of the nHAp-nanocarbon composites is induced mainly by the nHAp nanoparticles themselves. Only these provide the required bioinspired surface to trigger the growth of apatite under standard in vitro conditions. However, the characteristics of the nHAp nanoparticles, which in turn depend on the carbon support as discussed in Section 3.2, influence the apatite formation process. This affects their growth time, as well as the chemical composition and integration of the overall $(\mathrm{nHAp}+\mathrm{nAp})$ system on the respective carbon support. The observation that the nHAp integration on the nanocarbon support changes during the transient stages of 
bioactivity, and even may be substantially weakened in case apatite nanoparticles are allowed to form at the respective interface, has important implications for the rational synthesis design of nHAp-nanocarbon composite materials and elaboration of meaningful mechanical testing protocols.

\section{Conclusions}

Non stoichiometric nanocrystalline hydroxyapatite (nHAp) with a composition similar to natural bone was grown by a wet-chemical precipitation route on carbon nanotubes with different degrees of oxygen functionalities and on graphene oxide resulting in corresponding composite materials. Functionalization degree and morphology of CNTs and GO appear as critical parameters controlling the shape and crystallinity of the selfassembled nHAp nanoparticles. Crystalline nHAp nanoparticles express a rod-like morphology by establishing $\mathrm{O}-\mathrm{Ca}-\mathrm{O}$ interactions between calcium atoms from the crystallographic (300) planes of hydroxyapatite and oxygen atoms from functional groups located either at the sidewall of oxidized carbon nanotubes or at the basal plane of graphene oxide. The nHAp nanorods are intimately integrated on the respective oxidized carbon surface and in case of GO can form a dense coating layer. In vitro bioactivity of the resulting composites exhibits a fast apatite biomineralization process, induced by the presence of nHAp. Depending on the crystalline size and the dispersion degree of nHAp nanoparticles the bioactivity is accompanied by a stepwise ionexchange mechanism leading to a remineralization of the nAp nanoparticles through inclusion of $\mathrm{PO}_{4}{ }^{3-}$ and $\mathrm{CO}_{3}{ }^{2-}$ by ion exchange. Importantly, it appears that during the stage of bioactivity the integration degree of nHAp nanoparticles on the carbon support material is altering with time. Our findings on the mutual dependence of integration and bioactivity are of a more generic interest beyond the studied case. They underline the potential of graphene oxide as suitable carbon support material, the value of X-ray 
photoelectron spectroscopy (XPS) for evaluating the integration degree and, overall, emphasize the importance of testing novel biomaterials under physiological conditions. The obtained results are of critical importance for the design of bone implants and tissue engineering applications.

\section{Acknowledgements}

Financial supports from Spanish Ministry MINECO (project MAT2010-15026), CSIC (project 201080E123, PhD grant JAEPre09-01155 for J.D.N.), and Regional Government of Aragon and European Social Fund DGA-ESF (T66 Grupo Consolidado), the EU COST network MP0901 NanoTP and the EU $7^{\text {th }}$ Framework Program under Grant Agreement 312483-ESTEEM2 (Integrated Infrastructure Initiative - I3) are gratefully acknowledged.

\section{Appendix A. Supplementary data}

Supplementary data related to this article can be found at http://dx.doi.org/10.1016/j.carbon.2014.XX.XXX.

\section{References}

[1] Sadat-Shojai M, Khorasani M-T, Dinpanah-Khoshdargi E, Jamshidi A. Synthesis methods for nanosized hydroxyapatite with diverse structures. Acta Biomater. 2013;9(8):7591-621.

[2] O'Brien FJ. Biomaterials \&amp; scaffolds for tissue engineering. Mater. Today. 2011;14(3):88-95.

[3] Vallet-Regí M, González-Calbet JM. Calcium phosphates as substitution of bone tissues. Prog Solid State Chem. 2004;32:1-31. 
[4] White AA, Best SM, Kinloch IA. Hydroxyapatite-Carbon Nanotube Composites for Biomedical Applications: A Review. Int J Appl Ceramic Technol. 2007;4(1):1-13. [5] Saffar KP, Arshi AR, JamilPour N, Najafi AR, Rouhi G, Suda L. A cross-linking model for estimating Young's modulus of artificial bone tissue grown on carbon nanotube scaffold. J Biomed Mater Res A. 2010;94A:594-602.

[6] Shen ZJ, Adolfsson E, Nygren M, Gao L, Kawaoka H, Niihara K. Dense Hydroxyapatite-Zirconia Ceramic Composites with High Strength for Biological Applications. Adv Mater. 2001;13(3):214-6.

[7] Li H, Khor KA, Cheang P. Titanium dioxide reinforced hydroxyapatite coatings deposited by high velocity oxy-fuel (HVOF) spray. Biomaterials. 2002;23(1):85-91. [8] Li J, Fartash B, Hermansson L. Hydroxyapatite_-alumina composites and bonebonding. Biomaterials. 1995;16(5):417-22.

[9] Zhou S, Zheng X, Yu X, Wang J, Weng J, Li X, et al. Hydrogen Bonding Interaction of Poly(d,1-Lactide)/hydroxyapatite Nanocomposites. Chem Mater. 2006;19(2):247-53.

[10] Velayudhan S, Anilkumar TV, Kumary TV, Mohanan PV, Fernandez AC, Varma HK, et al. Biological evaluation of pliable hydroxyapatite-ethylene vinyl acetate copolymer composites intended for cranioplasty. Acta Biomater. 2005;1(2):201-9.

[11] Fang L, Leng Y, Gao P. Processing and mechanical properties of HA/UHMWPE nanocomposites. Biomaterials. 2006;27(20):3701-7.

[12] Lahiri D, Ghosh S, Agarwal A. Carbon nanotube reinforced hydroxyapatite composite for orthopedic application: A review. Mater Sci Eng: C. 2012;32(7):1727-58. [13] Dresselhaus MS, Dresselhaus G, Avouris P, eds. Carbon Nanotubes - Synthesis, Structure, Properties, and Applications. Berlin: Springer 2001. 
[14] Siegel RW, Chang SK, Ash BJ, Stone J, Ajayan PM, Doremus RW, et al. Mechanical behavior of polymer and ceramic matrix nanocomposites. Scr Mater. 2001;44(8-9):2061-4.

[15] Balani K, Chen Y, Harimkar SP, Dahotre NB, Agarwal A. Tribological behavior of plasma-sprayed carbon nanotube-reinforced hydroxyapatite coating in physiological solution. Acta Biomater. 2007;3(6):944-51.

[16] Chen Y, Zhang YQ, Zhang TH, Gan CH, Zheng CY, Yu G. Carbon nanotube reinforced hydroxyapatite composite coatings produced through laser surface alloying. Carbon. 2006;44(1):37-45.

[17] Chen Y, Zhang TH, Gan CH, Yu G. Wear studies of hydroxyapatite composite coating reinforced by carbon nanotubes. Carbon. 2007;45(5):998-1004.

[18] Lahiri D, Singh V, Keshri AK, Seal S, Agarwal A. Carbon nanotube toughened hydroxyapatite by spark plasma sintering: Microstructural evolution and multiscale tribological properties. Carbon. 2010;48(11):3103-20.

[19] Xu JL, Khor KA, Sui JJ, Chen WN. Preparation and characterization of a novel hydroxyapatite/carbon nanotubes composite and its interaction with osteoblast-like cells. Mater Sci Eng: C. 2009;29(1):44-9.

[20] Balani K, Anderson R, Laha T, Andara M, Tercero J, Crumpler E, et al. Plasmasprayed carbon nanotube reinforced hydroxyapatite coatings and their interaction with human osteoblasts in vitro. Biomaterials. 2007;28(4):618-24.

[21] Harrison BS, Atala A. Carbon nanotube applications for tissue engineering. Biomaterials. 2007;28(2):344-53.

[22] Zanello LP, Zhao B, Hu H, Haddon RC. Bone Cell Proliferation on Carbon Nanotubes. Nano Letters. 2006;6(3):562-7. 
[23] Nayak TR, Jian L, Phua LC, Ho HK, Ren Y, Pastorin G. Thin Films of Functionalized Multiwalled Carbon Nanotubes as Suitable Scaffold Materials for Stem Cells Proliferation and Bone Formation. ACS Nano. 2010;4(12):7717-25.

[24] Geim AK, Novoselov KS. The rise of graphene. Nature Materials. 2007;6(3):18391.

[25] Zhang L, Liu W, Yue C, Zhang T, Li P, Xing Z, et al. A tough graphene nanosheet/hydroxyapatite composite with improved in vitro biocompatibility. Carbon. 2013;61(0):105-15.

[26] Zhou H, Lee J. Nanoscale hydroxyapatite particles for bone tissue engineering. Acta Biomater. 2011;7(7):2769-81.

[27] Chang Y, Yang S-T, Liu J-H, Dong E, Wang Y, Cao A, et al. In vitro toxicity evaluation of graphene oxide on A549 cells. Toxicol Letters. 2011;200(3):201-10. [28] Xun X, Liu Z, Welsher K, Robinson JT, Goodwin A, Zaric S, et al. NanoGraphene Oxide for Cellular Imaging and Drug Delivery. Nano Res. 2008;1:203-12. [29] Nayak TR, Andersen H, Makam VS, Khaw C, Bae S, Xu X, et al. Graphene for Controlled and Accelerated Osteogenic Differentiation of Human Mesenchymal Stem Cells. ACS Nano. 2011;5(6):4670-8.

[30] Aryal S, Bahadur KCR, Dharmaraj N, Kim K-W, Kim HY. Synthesis and characterization of hydroxyapatite using carbon nanotubes as a nano-matrix. Scripta Mater. 2006;54(2):131-5.

[31] Liao S, Xu G, Wang W, Watari F, Cui F, Ramakrishna S, et al. Self-assembly of nano-hydroxyapatite on multi-walled carbon nanotubes. Acta Biomater. 2007;3(5):66975. 
[32] Lei T, Wang L, Ouyang C, Li N-F, Zhou L-S. In Situ Preparation and Enhanced Mechanical Properties of Carbon Nanotube/Hydroxyapatite Composites. Int J Appl Ceramic Technol. 2011;8(3):532-9.

[33] Xiao Y, Gong T, Zhou S. The functionalization of multi-walled carbon nanotubes by in situ deposition of hydroxyapatite. Biomaterials. 2010;31(19):5182-90.

[34] Beuvelot J, Bergeret C, Mallet R, Fernandez V, Cousseau J, Félix BM, et al. In vitro calcification of chemically functionalized carbon nanotubes. Acta Biomater. 2010;6(10):4110-7.

[35] Zhao L, Gao L. Novel in situ synthesis of MWNTs-hydroxyapatite composites. Carbon. 2004;42(2):423-6.

[36] Li M, Wang Y, Liu Q, Li Q, Cheng Y, Zheng Y, et al. In situ synthesis and biocompatibility of nano hydroxyapatite on pristine and chitosan functionalized graphene oxide. J Mater Chem B. 2013;1(4):475-84.

[37] Liu Y, Huang J, Li H. Synthesis of hydroxyapatite-reduced graphite oxide nanocomposites for biomedical applications: oriented nucleation and epitaxial growth of hydroxyapatite. J Mater Chem B. 2013;1(13):1826-34.

[38] Liu H, Xi P, Xie G, Shi Y, Hou F, Huang L, et al. Simultaneous Reduction and Surface Functionalization of Graphene Oxide for Hydroxyapatite Mineralization. J Phys Chem C. 2012;116(5):3334-41.

[39] Baradaran S, Moghaddam E, Basirun WJ, Mehrali M, Sookhakian M, Hamdi M, et al. Mechanical properties and biomedical applications of a nanotube hydroxyapatitereduced graphene oxide composite. Carbon. 2014;69(0):32-45.

[40] Fan Z, Wang J, Wang Z, Ran H, Li Y, Niu L, et al. One-pot synthesis of graphene/hydroxyapatite nanorod composite for tissue engineering. Carbon. 2014;66(0):407-16. 
[41] Shin US, Yoon I-K, Lee G-S, Jang W-C, Knowles JC, Kim H-W. Carbon Nanotubes in Nanocomposites and Hybrids with Hydroxyapatite for Bone Replacements. J Tissue Eng. 2011;2011:674287.

[42] Hummers WS, Offeman RE. Preparation of Graphitic Oxide. J Am Chem Soc. 1958;80(6):1339.

[43] Li D, Müller MB, Gilje S, Kaner RB, Wallace GG. Processable aqueous dispersions of graphene nanosheets. Nat Nanotechnol. 2008;3(2):101-5.

[44] Vallés C, David Núñez J, Benito AM, Maser WK. Flexible conductive graphene paper obtained by direct and gentle annealing of graphene oxide paper. Carbon. 2012;50(3):835-44.

[45] Vallés C, Jiménez P, Muñoz E, Benito AM, Maser WK. Simultaneous Reduction of Graphene Oxide and Polyaniline: Doping-Assisted Formation of a Solid-State Charge-Transfer Complex. J Phys Chem C. 2011;115:10468-74.

[46] Kokubo T, Takadama H. How useful is SBF in predicting in vivo bone bioactivity? Biomaterials. 2006;27(15):2907-15.

[47] Implants for surgery - In vitro evaluation for apatite-forming ability of implant materials. ISO 23317. Geneva: Int Org Standard (ISO) 2007:13.

[48] Figueiredo JL, Pereira MFR. The role of surface chemistry in catalysis with carbons. Catalysis Today. 2010;150(1,2):2-7.

[49] Martínez MT, Callejas MA, Benito AM, Cochet M, Seeger T, Ansón A, et al. Sensitivity of single wall carbon nanotubes to oxidative processing: structural modification, intercalation and functionalisation. Carbon. 2003;41(12):2247-56. [50] Gao W, Alemany LB, Ci L, Ajayan PM. New insights into the structure and reduction of graphite oxide. Nat Chem. 2009;1(5):403-8. 
[51] Loh KP, Bao Q, Eda G, Chhowalla M. Graphene oxide as a chemically tunable platform for optical applications. Nat Chem. 2010;2(12):1015-24.

[52] Prevèy PS. X-ray diffraction characterization of crystallinity and phase composition in plasma-sprayed hydroxylapatite coatings. J Therm Spray Technol. 2000;9(3):369-76.

[53] Low I-M. Depth-Profiling of Crystal Structure, Texture, and Microhardness in a Functionally Graded Tooth Enamel. J Am Ceramic Soc. 2004;87(11):2125-31.

[54] Corno M, Rimola A, Bolis V, Ugliengo P. Hydroxyapatite as a key biomaterial: quantum-mechanical simulation of its surfaces in interaction with biomolecules. Phys Chem Chem Phys. 2010;12:6309-632.

[55] Acik M, Mattevi C, Gong C, Lee G, Cho K, Chhowalla M, et al. The Role of Intercalated Water in Multilayered Graphene Oxide. ACS Nano. 2010;4(10):5861-8.

[56] Posner AS, Perloff A, Diorio AF. Refinement of the hydroxyapatite structure. Acta Cryst. 1958; 11:308.

[57] Rivera-Muñoz EM. Hydroxyapatite-Based Materials: Synthesis and Characterization. In: Reza-Fazel-Rezai, ed. Medical Engineering - Frontiers and Challenges. Rijeka, Croatia: InTech publisher 2011:75-98.

[58] Nakada H, Numata Y, Sakae T, Kimura-Suda H, Tanimoto Y, Saeki H, et al. Changes in bone qualitiy associated with the mineralizaiton ofnew bone formed around implants - using XPS, Polarized Microscopy, and FTIR imaging -. J Hard Tissue Biol. 2010;19(2):101-10.

[59] Demri B, Muster D. XPS study of some calcium compounds. J Mater Proces Technol. 1995;55(3,4):311-4. 
[60] Chang MC, Tanaka J. XPS study for the microstructure development of hydroxyapatite-collagen nanocomposites cross-linked using glutaraldehyde. Biomaterials. 2002;23(18):3879-85.

[61] Sakamoto H, Hirohashi Y, Saito H, Doi H, Tsutsumi Y, Suzuki Y, et al. Effect of active hydroxyl groups on the interfacial bond strength of titanium with segmented polyurethane through $\gamma$-mercapto propyl trimethoxysilane. Dental Mater J. 2008;27(1):81-92.

[62] Kim H-M, Himeno T, Kokubo T, Nakamura T. Process and kinetics of bonelike apatite formation on sintered hydroxyapatite in a simulated body fluid. Biomaterials. 2005;26(21):4366-73. 